\title{
Galactic abundance gradients from Cepheids
}

\section{$\alpha$ and heavy elements in the outer disk ${ }^{\star} \star \star \star$}

\author{
B. Lemasle ${ }^{1,2}$, P. François ${ }^{3,4}$, K. Genovali ${ }^{5}$, V. V. Kovtyukh 6 , G. Bono ${ }^{5,7}$, L. Inno ${ }^{5,13}$, C. D. Laney ${ }^{8,9}$, L. Kaper ${ }^{1}$, \\ M. Bergemann ${ }^{10}$, M. Fabrizio ${ }^{11}$, N. Matsunaga ${ }^{12}$, S. Pedicelli ${ }^{5}$, F. Primas ${ }^{13}$, and M. Romaniello ${ }^{13}$ \\ 1 Astronomical Institute Anton Pannekoek, Science Park 904, PO Box 94249, 1090 GE Amsterdam, The Netherlands \\ e-mail: B. J.P.Lemasle@uva.nl \\ 2 Kapteyn Astronomical Institute, Landleven 12, 9747 AD Groningen, The Netherlands \\ 3 GEPI - Observatoire de Paris, 64 avenue de l'Observatoire, 75014 Paris, France \\ ${ }^{4}$ UPJV - Université de Picardie Jules Verne, 80000 Amiens, France \\ 5 Dipartimento di Fisica, Università di Roma Tor Vergata, via della Ricerca Scientifica 1, 00133 Rome, Italy \\ 6 Astronomical Observatory of Odessa National University, and Isaac Newton Institute of Chile, Odessa branch, Shevchenko Park, \\ 65014 Odessa, Ukraine \\ 7 INAF - Osservatorio Astronomico di Roma, via Frascati 33, Monte Porzio Catone, Rome, Italy \\ 8 South African Astronomical Observatory, PO Box 9, Observatory 7935, South Africa \\ 9 Department of Physics and Astronomy, Western Kentucky University, 1906 College Heights Blvd, Bowling Green, \\ KY 42101-1077, USA \\ 10 Max Planck Institute for Astrophysics, Karl-Schwarzschild-Str. 1, 85741 Garching bei Munchen, Germany \\ 11 INAF - Osservatorio Astronomico Collurania, via M. Maggini, 64100 Teramo, Italy \\ 12 Department of Astronomy, School of Science, The University of Tokyo, 7-3-1 Hongo, Bunkyo-ku, 113-0033 Tokyo, Japan \\ 13 European Southern Observatory, Karl-Schwarzschild-Str. 2, 85748 Garching bei Munchen, Germany
}

Received 21 June 2013 / Accepted 28 July 2013

\begin{abstract}
Context. Galactic abundance gradients set strong constraints to chemo-dynamical evolutionary models of the Milky Way. Given the period-luminosity relations that provide accurate distances and the large number of spectral lines, Cepheids are excellent tracers of the present-day abundance gradients.

Aims. We want to measure the Galactic abundance gradient of several chemical elements. While the slope of the Cepheid iron gradient did not vary much from the very first studies, the gradients of the other elements are not that well constrained. In this paper we focus on the inner and outer regions of the Galactic thin disk.

Methods. We use high-resolution spectra (FEROS, ESPADONS, NARVAL) to measure the abundances of several light (Na, Al), $\alpha(\mathrm{Mg}, \mathrm{Si}, \mathrm{S}, \mathrm{Ca})$, and heavy elements $(\mathrm{Y}, \mathrm{Zr}, \mathrm{La}, \mathrm{Ce}, \mathrm{Nd}, \mathrm{Eu})$ in a sample of 65 Milky Way Cepheids. Combining these results with accurate distances from period-Wesenheit relations in the near-infrared enables us to determine the abundance gradients in the Milky Way.

Results. Our results are in good agreement with previous studies on either Cepheids or other tracers. In particular, we confirm an upward shift of $\approx 0.2 \mathrm{dex}$ for the $\mathrm{Mg}$ abundances, as has recently been reported. We also confirm the existence of a gradient for all the heavy elements studied in the context of a local thermodynamic equilibrium analysis. However, for Y, Nd, and especially La, we find lower abundances for Cepheids in the outer disk than reported in previous studies, leading to steeper gradients. This effect can be explained by the differences in the line lists used by different groups.

Conclusions. Our data do not support a flattening of the gradients in the outer disk, in agreement with recent Cepheid studies and chemo-dynamical simulations. This is in contrast to the open cluster observations but remains compatible with a picture where the transition zone between the inner disk and the outer disk would move outward with time.
\end{abstract}

Key words. stars: abundances - supergiants - stars: variables: Cepheids - Galaxy: abundances - Galaxy: evolution - Galaxy: disk

\footnotetext{
$\star$ Based on observations obtained at the Canada-France-Hawaii Telescope (CFHT), which is operated by the National Research Council of Canada, the Institut National des Sciences de l'Univers of the Centre National de la Recherche Scientifique of France, and the University of Hawaii. Based on observations collected with FEROS at the European Southern Observatory (La Silla, Chile) under proposal ID: 60.A-9120. Based on observations obtained at the Telescope Bernard Lyot (USR5026) operated by the Observatoire Midi-Pyrénées and the Institut National des Science de l'Univers of the Centre National de la Recherche Scientifique of France. The data of NARVAL were reduced using the data reduction software Libre-ESpRIT, written and provided by Donati from the LATT (Observatoire Midi-Pyrénées).
}

\section{Introduction}

The distribution of the elements along the disk of galaxies is influenced by the star formation history (SFH), accretion events, radial flows of gas, and radial mixing of stars. It usually follows a radial gradient. Radial abundance gradients are therefore used to constrain models describing the formation and evolution of galaxies, in particular of the Milky Way.

\footnotetext{
$\star \star$ Appendices are available in electronic form at http://www . aanda.org
} 
Different observational tracers have been used to derive the Galactic abundance gradients: HII regions, O/B-type stars, Cepheids, open clusters and planetary nebulae (PNe). Because the nature of these objects is different, it allows us to trace different elements and age groups, paving the way for time evolution studies that are still in their first faltering steps.

Compared to the other tracers, Cepheids present several advantages: i) as famous primary distance indicators, they provide very accurate distances; ii) they are bright stars and can thus still be observed at large distances from the Sun; iii) they are cool supergiants that present a large set of well-defined absorption lines. Therefore accurate abundances of many elements can be determined. On the other hand, the population of Galactic Cepheids is still relatively poorly sampled, and the young age of these stars ( $<200$ Myr, Bono et al. 2005) allows us to study only the presentday gradient. As Cepheids are pulsating variable stars, one could wonder whether the phase of observation would influence the abundance determination. However, Luck \& Andrievsky (2004), Kovtyukh et al. (2005a), Andrievsky et al. (2005), and Luck et al. (2008) have demonstrated in a series of papers that results are extremely stable throughout the period, whatever the period of the star.

Since the early works of Harris $(1981,1984)$, the determinations of the Galactic iron gradient from Cepheids have been remarkably consistent: Andrievsky et al. (2002a,b,c), Luck et al. (2003, 2006, 2011), Andrievsky et al. (2004), Kovtyukh et al. (2005b), Luck \& Lambert (2011), Lemasle et al. (2007, 2008), and Pedicelli et al. $(2009,2010)$ all report a value close to $-0.06 \mathrm{dex} / \mathrm{kpc}$. Cepheid abundances have also been derived by Yong et al. (2006); Usenko et al. (2011a,b).

These values are in good agreement with studies based on other tracers, even if a direct comparison between them is quite difficult: as already mentioned, different objects trace different elements and different ages. The abundances in HII regions can be derived from radio recombination lines, collisionally excited lines, or optical recombination lines that lead to somewhat discrepant abundances (the same also holds for PNe). Therefore the $[\mathrm{O} / \mathrm{H}]$ gradients span quite a large range of values, which is between $-0.030 \mathrm{dex} / \mathrm{kpc}$ and $-0.08 \mathrm{dex} / \mathrm{kpc}$ if we consider only the recent determinations (e.g., Simpson et al. 1995; Afflerbach et al. 1996, 1997; Deharveng et al. 2000; Quireza et al. 2006b; Rudolph et al. 2006; Balser et al. 2011).

The gradients obtained with O-B1 stars vary between -0.03 and -0.07 dex/kpc (e.g., Smartt \& Rolleston 1997; Gummersbach et al. 1998; Daflon \& Cunha 2004b; Rolleston et al. 2000). As O-B1 stars are younger than $10 \mathrm{Myr}$, they trace the present-day gradient even more closely than Cepheids.

There are numerous abundance studies of open clusters from different groups: Bragaglia et al. (2008), Carraro et al. (2007), Chen et al. (2003), Friel et al. (2002, 2010), Jacobson et al. (2008, 2009, 2011a,b), Magrini et al. (2009a, 2010), Pancino et al. (2010), Sestito et al. (2006, 2007), Yong et al. (2005, 2012). They all reach the same conclusion: a linear gradient of approximately $-0.06 \mathrm{dex} / \mathrm{kpc}$ extending quite far into the outer disk, and a flattening at a level of $[\mathrm{Fe} / \mathrm{H}] \approx-0.3 \mathrm{dex}$, somewhere between 10 and $14 \mathrm{kpc}$. The only exception is Sestito et al. (2008), who report a larger slope of $-0.17 \mathrm{dex} / \mathrm{kpc}$ in the inner part of the disk.

Radial $[\mathrm{O} / \mathrm{H}]$ gradients from PNe have values generally comprised between $-0.02 \mathrm{dex} / \mathrm{kpc}$ and $-0.06 \mathrm{dex} / \mathrm{kpc}$ (Maciel et al. 1999; Costa et al. 2004; Henry et al. 2004; Perinotto \& Morbidelli 2006; Henry et al. 2010). Pottasch \& Bernard-Salas (2006) obtain better accuracies for their abundances because they measure transitions both in the visible and in the infrared.
However, their sample does not extend beyond $\approx 10 \mathrm{kpc}$ (where the most metal-poor PNe are found), which might be why they report a stronger gradient of $-0.085 \mathrm{dex} / \mathrm{kpc}$. In contrast to the other studies, Stanghellini et al. (2006) find no evidence of a gradient.

The time evolution of the metallicity gradients is still very controversial: from PNe, Maciel et al. (2003) found a flattening of the gradient with time, while Stanghellini \& Haywood (2010) report instead a steepening of the gradient and Henry et al. (2010) found no evolution with time. Most studies dividing open clusters into age bins report a flattening with time. When combining several tracers, Maciel \& Costa (2009) again found a flattening with time, while Magrini et al. (2009a) report only marginal evidence of gradients flattening with time in the $7-12 \mathrm{kpc}$ range. From a comparison between Open Clusters and Cepheids, Yong et al. (2012) report a flattening of the gradient with time in the [5-12] kpc range for all the elements considered in their study.

However, a simple, linear gradient may not be the better way to describe the distribution of the elements in the Galactic disk. From Cepheids, Andrievsky et al. (2002b), Pedicelli et al. (2009, 2010), and Genovali et al. (2013) found a steeper gradient in the very inner disk $(\leq 7 \mathrm{kpc})$. We have already mentioned that most of the studies based on open clusters find a flatter gradient in the outer disk. In this region, the situation is much less clear when considering other objects: e.g., Vilchez \& Esteban (1996, HII regions) and Costa et al. (2004, PNe) report flat gradients in the outskirts of the Milky Way, while other authors using the same tracers do not. From Cepheids, a flattening gradient seemed well established (Andrievsky et al. 2002c, 2004; Luck et al. 2003; Lemasle et al. 2008), but the recent addition of outer disk Cepheids in the sample (Luck et al. 2011; Luck \& Lambert 2011) weakens this hypothesis. A flattening gradient is also seen in external galaxies, (e.g., Bresolin et al. 2009a). Moreover, Yong et al. (2006) brought forward an interesting feature: the flat structure in the outer disk may occur with two different basement values, one at -0.5 dex and a second one as low as -0.8 dex, possibly related to a merger event.

In addition, Twarog et al. (1997) suggested that instead of a break in the slope the radial metallicity gradient could experience an abrupt drop of the order of $0.2-0.3$ dex around $10 \mathrm{kpc}$. This feature was also proposed in other studies (e.g., Daflon \& Cunha 2004b; Andrievsky et al. 2004). From the open cluster point of view, it is still not clear whether this feature is real or only due to a too coarse sampling, despite the rapidly growing number of studies investigating this zone. Whether the metallicity distribution takes the shape of a step or more simply of a change in the slope, the exact location of the transition could be a bit further out in the disk and depend on the cluster ages (e.g., Jacobson et al. 2011b). For Cepheids, the step was probably an artifact due to a sampling effect (Lemasle et al. 2008; Luck et al. 2011; Luck \& Lambert 2011). Such a feature in the outer disk is generally supported by theoretical works invoking variations of the gas density, the presence of a bar, or the consequence of long-lived spiral structures (Scarano \& Lépine 2013).

Negative abundance gradients, comparable to those of the Milky Way, have also been reported in external spiral galaxies: e.g., M31 (Sanders et al. 2010), M33 (Barker et al. 2007; Magrini et al. 2009b), M83 (Bresolin et al. 2009a), NGC 300 (Kudritzki et al. 2008; Vlajić et al. 2009; Bresolin et al. 2009b), NGC 7793 (Vlajić et al. 2009). In most cases the gradient also flattens in the outer disk.

The chemical enrichment of the Galaxy was first studied by pure chemical evolution models, most of them based on the 
so-called inside-out scenario (e.g., Matteucci \& François 1989; Chiappini et al. 1997, 2001; Prantzos \& Boissier 2000; Mollá \& Díaz 2005; Fu et al. 2009) in which the Milky Way primarily forms during two episodes of gas infall: the first one giving birth to the halo and the bulge and the second one producing the disk. During the second infall, the pristine gas accumulates more slowly and preferentially in the inner parts of the disk, leading to a faster chemical enrichment of these regions. Therefore a negative abundance gradient naturally arises within the Galactic disk. In particular, the model from Cescutti et al. (2007) computes radial gradients for numerous elements and closely reproduces Cepheid-based observations. Radial flows of gas (up to a few $\mathrm{km} \mathrm{s}^{-1}$, see Lacey \& Fall 1985; Portinari \& Chiosi 2000; Spitoni $\&$ Matteucci 2011) must also be taken into account to properly fit the observational data, in particular to recover the gas density distribution. Radial gas flows also enable steep enough gradients in the models (Spitoni \& Matteucci 2011; but see Fu et al. 2013) to be obtained, although the alternative explanation of a variable efficiency in the star formation rate could have a similar effect (Colavitti et al. 2008, and references therein).

More recently, dynamical aspects have also been taken into account through $N$-body/smoothed particle hydrodynamics (SPH), semi-analytical models, or cosmological simulations of galactic disks (e.g., Stinson et al. 2010; Rahimi et al. 2011; Kobayashi \& Nakasato 2011; Few et al. 2012, for the latest ones). Wiersma et al. (2011) systematically investigated the impact on the outcome when, not specifically looking for Milky Way analogs, they varied a large number of physical processes. On the other hand, Pilkington et al. (2012) compared different sets of simulations and two classical chemical evolution models, all fine-tuned to resemble the Milky Way. All of the models were able to reproduce the present-day gradient, but following different paths.

The radial migration of stars along a Galactic radius has recently been recognized as a key process to understanding the evolution of gradients with time. Several mechanisms have been proposed to explain the radial mixing:

- the position of stars around their birth radii shows an increased spread due to scattering by molecular clouds, which increases their epicyclic energy ("blurring", Spitzer \& Schwarzschild 1953; Binney \& Tremaine 2008);

- the orbit of stars is moved inward or outward either by resonant scattering by the transient spiral structure (Sellwood \& Binney 2002; Roškar et al. 2008a,b; Schönrich \& Binney 2009), or by the resonance overlap of the bar and spiral structure (Minchev \& Famaey 2010; Minchev et al. 2011). This mechanism is referred to as "churning" and implies a change in the angular momentum of the star;

- radial mixing can also be caused by the perturbation induced by a low-mass orbiting satellite (Quillen et al. 2009; Bird et al. 2012) or a satellite bombardment (Bird et al. 2012).

There were early models combining dynamics and chemical evolution (e.g., François \& Matteucci 1993). Schönrich \& Binney (2009) were the first to incorporate the recent results on radial migration of stars in chemical evolution models. Very recently, Minchev et al. (2013) presented new Milky Way models, where the chemical evolution is merged with disk numerical simulations. Each particle in their simulations represents only one star, while the $N$-body simulations with the highest resolution and an improved treatment of the chemistry (e.g., Bird et al. 2013) have particles of $\approx 10^{4} M_{\odot}$. Among other results, they found that radial migration affects the old stars more strongly.
Radial migration in the disk is at the origin of azimuthal variations in the metallicity distribution of its old stars (Di Matteo et al. 2013).

In this paper we investigate the shape of the gradient for 11 elements and focus on the outer disk. The paper is organized as follows: in Sect. 2, we briefly recall the observations and data analysis, and in Sect. 3 we describe the distances of the Cepheids based on near-infrared (NIR) photometry. Results are presented and discussed in Sect. 4 (abundances) and Sect. 5 (gradients). Section 6 summarizes our findings.

\section{Observations and method}

\subsection{Data}

Our study is based on the same high-resolution spectra (ESPaDOnS: 25 stars, FEROS: 40 stars, Narval@TBL: 1 star) as in Lemasle et al. $(2007,2008)$ and Pedicelli et al. (2010), where we determined the atmospheric parameters and iron abundances in order to study the iron gradient across the Galactic disk. The signal to noise ratio $(\mathrm{S} / \mathrm{N})$ ranges from 40 to 140 , with most of the spectra having $S / N \simeq 100$.

\subsection{Atmospheric parameters}

We used the atmospheric parameters previously derived in the papers quoted above. For this reason, we only briefly summarize the method here. Because Cepheids are pulsating variable stars, it is very unlikely to obtain simultaneous photometric measurements. Therefore the atmospheric parameters have to be determined exclusively from spectroscopic information. To accurately determine $T_{\text {eff }}$, which is critical in the process of abundance determination, we used the line-depth ratios method described in Kovtyukh \& Gorlova (2000) and Kovtyukh (2007). This method has been long proven to give accurate temperatures and can also be double-checked because lines with both high and low $\chi_{\text {ex }}$ values have to properly fit the curve of growth. The surface gravity, $\log g$, and the microturbulent velocity, $v_{\mathrm{t}}$, were determined by a classical spectroscopic analysis: we imposed i) the ionization balance between Fe I and Fe II with the help of the curve of growth and ii) that the Fe I abundance depends neither on line strength nor on the excitation potential. It should be noted here that Andrievsky and collaborators use a slightly modified version of the canonical analysis in order to avoid a possible influence of non local thermodynamic equilibrium (NLTE) effects on Fe I lines (Kovtyukh \& Andrievsky 1999): surface gravities and microturbulent velocities are derived from Fe II lines instead of Fe I lines. However, the two methods lead to differences that are typically equal to or less than 0.10 dex for the $[\mathrm{Fe} / \mathrm{H}]$ abundances.

\subsection{Line list}

Some usual lines in stellar spectroscopic analysis cannot be used to determine the chemical composition of Cepheids as they are much too wide in supergiants. For instance, the $\mathrm{Mg} \mathrm{I}$ line at $5528.41 \AA$ as well as the Ba II lines at 6141.73 and $6496.91 \AA$ have equivalent widths (EW) larger than $250 \mathrm{~m} \AA$ in most of the stars of our sample. This is the reason why the first study of barium in Cepheids (which takes into account NLTE effects) appeared only very recently (Andrievsky et al. 2013). Surprisingly, it is also the first large study of the barium abundance across the Galactic disk. Moreover, Cepheids span a very wide range of $T_{\text {eff }}, \log g$, and $v_{\mathrm{t}}$ along the pulsation cycle. In particular, $T_{\text {eff }}$ can span a range larger than $1000 \mathrm{~K}$. As abundances should ideally be derived from the same set of lines for the whole sample, 
one wants to discard lines that would disappear or become too strong at the edges of the $T_{\text {eff }}$ range. The same outcome applies to the other atmospheric parameters. Finally, in the phases with the lowest $T_{\text {eff }}$ and $\log g$, some parts of the spectrum, especially toward the blue, are so crowded that line blending makes it almost impossible to locate the continuum, preventing us from measuring lines in those parts of the spectrum.

In order to build a proper line list, we selected a dozen of our spectra with a good $\mathrm{S} / \mathrm{N}$ that covered the atmospheric parameter space (including $[\mathrm{Fe} / \mathrm{H}]$ ) as much as possible. From those spectra, we picked up the lines fulfilling all the aforementioned criteria, namely, lines that are always present but never too strong, with no or limited blending, and with a continuum well defined around the line. Despite this careful selection, it could still happen that some of the lines were not measurable, either because the Cepheids were observed in a phase that was less favorable or more simply because we could only reach a limited S/N. For all the lines, we adopted the physical properties (oscillator strength, excitation potential) listed in the Vienna Atomic Line Database (VALD; Kupka et al. 1999; Ryabchikova et al. 1999). Our line list for the $\alpha$ and heavy elements can be found in Table A.1. The Fe I and Fe II lines that were used to determine the atmospheric parameters of the Cepheids are listed in Romaniello et al. (2008) and Pedicelli et al. (2010); the EW of iron lines were not remeasured here.

\subsection{Abundance determinations}

The atmospheric parameters are used as input for the MARCS models of Edvardsson et al. (1993). In this version of the MARCS models, the stellar atmosphere is described assuming a plane-parallel stratification, hydrostatic equilibrium, and local thermodynamic equilibrium (LTE). The EW of $\alpha$ and heavy elements were measured with a semi-interactive code (fitline, written by P. François) based on genetic algorithms from Charbonneau (1995) (see Lemasle et al. 2007 for details). We adopted the solar chemical abundances provided by Grevesse et al. (1996). For a given element, the final abundance of a star is estimated as the mean value of the abundances determined for each line of this element.

In order to test how the uncertainties on the atmospheric parameters of the star affect the final abundance results, we computed the abundances with over- or underestimated values of $T_{\text {eff }}( \pm 100 \mathrm{~K}), \log g( \pm 0.3 \mathrm{dex}), v_{\mathrm{t}}( \pm 0.5 \mathrm{dex})$, and $[\mathrm{Fe} / \mathrm{H}]$ $( \pm 0.12 \mathrm{dex})$. The sum in quadrature of these uncertainties on the atmospheric parameters gives uncertainties on the abundances that are listed in Table 1 (uncertainties of the order of 0.12 dex on $[\mathrm{Fe} / \mathrm{H}]($ Lemasle et al. 2008) leave the abundances unchanged and are therefore not included in Table 1). We repeated the exercise for two stars spanning the whole temperature range for Cepheids: SX Vel $\left(T_{\text {eff }}=6248 \mathrm{~K}\right)$ and SV Mon $\left(T_{\text {eff }}=4900 \mathrm{~K}\right)$

\section{Distances}

\subsection{Period-Wesenheit relations}

For a given star, the Wesenheit index (e.g., Madore 1982) is a linear combination of a selected magnitude and one of its related colors. By construction, the Wesenheit index is a reddening-free quantity. For instance, the Wesenheit index for $J$ and $K$ magnitudes $\mathrm{W}_{J K}$ is defined as

$W_{J K}=K-\frac{A_{K}}{E(J-K)} \times(J-K)$.

Compared to traditional period-luminosity(-color) relations, the period-Wesenheit $(\mathrm{P}-\mathrm{W})$ relations present several advantages.
Table 1. Top: error budget for SX Vel $\left(T_{\text {eff }}=6250 \mathrm{~K}, \log g=1.3 \mathrm{dex}\right.$, $\left.v_{\mathrm{t}}=2.8 \mathrm{~km} \mathrm{~s}^{-1},[\mathrm{Fe} / \mathrm{H}]=-0.2 \mathrm{dex}\right)$. Bottom: error budget for SV Mon $\left(T_{\text {eff }}=4900 \mathrm{~K}, \log g=0.50 \mathrm{dex}, v_{\mathrm{t}}=3.4 \mathrm{~km} \mathrm{~s}^{-1},[\mathrm{Fe} / \mathrm{H}]=-0.1 \mathrm{dex}\right)$.

\begin{tabular}{l|cccc}
\hline \hline Element & $\begin{array}{c}\Delta T_{\text {eff }} \\
(-100 \mathrm{~K}) \\
\text { dex }\end{array}$ & $\begin{array}{c}\Delta \log g \\
(-0.3 \text { dex }) \\
\text { dex }\end{array}$ & $\begin{array}{c}\Delta v_{\mathrm{t}} \\
(+0.5 \text { dex }) \\
\text { dex }\end{array}$ & $\begin{array}{c}\Delta[\mathrm{X} / \mathrm{H}] \\
\text { dex }\end{array}$ \\
\hline$[\mathrm{Al} / \mathrm{H}]$ & -0.04 & +0.02 & -0.02 & 0.05 \\
{$[\mathrm{Ca} / \mathrm{H}]$} & -0.07 & +0.03 & -0.09 & 0.12 \\
{$[\mathrm{Ce} / \mathrm{H}]$} & -0.07 & -0.08 & -0.13 & 0.17 \\
{$[\mathrm{Eu} / \mathrm{H}]$} & -0.06 & -0.08 & -0.04 & 0.11 \\
{$[\mathrm{La} / \mathrm{H}]$} & -0.07 & -0.07 & -0.03 & 0.11 \\
{$[\mathrm{Mg} / \mathrm{H}]$} & -0.06 & +0.02 & -0.08 & 0.11 \\
{$[\mathrm{Na} / \mathrm{H}]$} & -0.06 & +0.02 & -0.16 & 0.17 \\
{$[\mathrm{Nd} / \mathrm{H}]$} & -0.09 & -0.06 & -0.04 & 0.12 \\
{$[\mathrm{~S} / \mathrm{H}]$} & +0.00 & -0.04 & -0.11 & 0.12 \\
{$[\mathrm{Si} / \mathrm{H}]$} & -0.06 & +0.02 & -0.07 & 0.10 \\
{$[\mathrm{Y} / \mathrm{H}]$} & -0.05 & -0.09 & -0.05 & 0.12 \\
{$[\mathrm{Zr} / \mathrm{H}]$} & -0.04 & -0.09 & -0.06 & 0.12 \\
\hline & $\Delta T_{\text {eff }}$ & $\Delta \log g$ & $\Delta v_{\mathrm{t}}$ & $\Delta[\mathrm{X} / \mathrm{H}]$ \\
$\mathrm{Element}$ & $++100 \mathrm{~K})$ & $(+0.3 \mathrm{dex})$ & $(-0.5 \mathrm{dex})$ & \\
& dex & dex & dex & dex \\
\hline$[\mathrm{Al} / \mathrm{H}]$ & +0.06 & -0.03 & +0.09 & 0.12 \\
{$[\mathrm{Ca} / \mathrm{H}]$} & +0.07 & -0.02 & +0.03 & 0.08 \\
{$[\mathrm{Ce} / \mathrm{H}]$} & +0.00 & +0.13 & +0.04 & 0.14 \\
{$[\mathrm{Eu} / \mathrm{H}]$} & -0.01 & +0.13 & +0.10 & 0.17 \\
{$[\mathrm{La} / \mathrm{H}]$} & +0.02 & +0.13 & +0.14 & 0.19 \\
{$[\mathrm{Mg} / \mathrm{H}]$} & +0.05 & -0.02 & +0.13 & 0.14 \\
{$[\mathrm{Na} / \mathrm{H}]$} & - & - & - & - \\
{$[\mathrm{Nd} / \mathrm{H}]$} & +0.00 & +0.13 & +0.07 & 0.15 \\
{$[\mathrm{~S} / \mathrm{H}]$} & - & - & - & - \\
{$[\mathrm{Si} / \mathrm{H}]$} & +0.05 & +0.02 & +0.09 & 0.11 \\
{$[\mathrm{Y} / \mathrm{H}]$} & -0.01 & +0.14 & +0.13 & 0.19 \\
{$[\mathrm{Zr} / \mathrm{H}]$} & -0.01 & +0.12 & +0.20 & 0.24 \\
\hline & & & &
\end{tabular}

- They are almost linear over the entire period range;

- the slopes of P-W relations are almost independent of the metallicity;

- the dispersion of the P-W relations is significantly reduced compared to their corresponding period-luminosity (P-L) relations;

- P-W relations are reddening-free, whereas the use of classical $\mathrm{P}-\mathrm{L}$ relations requires the determination of the individual $E(B-V)$ for each Cepheid.

These characteristics of P-W relations have been predicted by theoretical pulsation models (e.g., Fiorentino et al. 2007; Bono et al. 2008). They have been supported from an observational point of view, in particular as far as the $\mathrm{P}-\mathrm{W}_{V I}$ is concerned, because a very large amount of data is already available in those bands (e.g., Fouqué et al. 2007; Madore \& Freedman 2009; Bono et al. 2010; Ngeow 2012, and references therein). The metallicity dependence of the optical $\mathrm{P}-\mathrm{W}_{V I}$ relations remains, however, controversial (Storm et al. (2011a,b), and Shappee et al. (2011); Gerke et al. (2011), but see also Majaess et al. (2011) for a discussion).

\subsection{Method}

New NIR P-W relations for Galactic Cepheids have been determined by Inno et al. (2013) for the $J, H$, and $K$ bands. Their slopes have been calibrated with Cepheids in the Large Magellanic Cloud and their zero-points with parallax distances from the Hubble Space Telescope available for Galactic Cepheids. In order to reduce the sources of uncertainties, first 
B. Lemasle et al.: Galactic abundance gradients from Cepheids

Table 2. Galactocentric distances and uncertainties for the Cepheids in our sample.

\begin{tabular}{ccccccccc}
\hline \hline Star & $\begin{array}{c}R_{\mathrm{G}} \\
\mathrm{kpc}\end{array}$ & $\begin{array}{c}\sigma-R_{\mathrm{G}} \\
\mathrm{kpc}\end{array}$ & Star & $\begin{array}{c}R_{\mathrm{G}} \\
\mathrm{kpc}\end{array}$ & $\begin{array}{c}\sigma-R_{\mathrm{G}} \\
\mathrm{kpc}\end{array}$ & Star & $\begin{array}{c}R_{\mathrm{G}} \\
\mathrm{kpc}\end{array}$ & $\begin{array}{c}\sigma-R_{\mathrm{G}} \\
\mathrm{kpc}\end{array}$ \\
\hline AA Gem & 11.454 & 0.459 & DR Vel & 8.054 & 0.439 & TX Mon & 11.790 & 0.452 \\
AD Gem & 10.662 & 0.455 & EK Mon & 9.960 & 0.453 & TY Mon & 11.180 & 0.451 \\
AD Pup & 10.588 & 0.434 & EZ Vel & 12.119 & 0.358 & TZ Mon & 11.183 & 0.451 \\
AH Vel & 8.074 & 0.450 & HW Pup & 13.554 & 0.436 & UX Car & 7.698 & 0.444 \\
AO Aur & 11.835 & 0.461 & 1 Car & 7.845 & 0.451 & UY Mon & 10.539 & 0.459 \\
AO CMa & 10.430 & 0.433 & MY Pup & 8.096 & 0.450 & UZ Sct & 5.309 & 0.448 \\
AP Pup & 8.234 & 0.449 & RS Ori & 9.470 & 0.453 & V340 Ara & 4.657 & 0.427 \\
AQ Car & 7.658 & 0.425 & RS Pup & 8.585 & 0.444 & V397 Car & 7.679 & 0.447 \\
AQ Pup & 9.472 & 0.436 & RY CMa & 8.796 & 0.450 & V495 Mon & 12.098 & 0.453 \\
AT Pup & 8.484 & 0.445 & RY Vel & 7.774 & 0.432 & V508 Mon & 10.714 & 0.452 \\
AV Sgr & 5.980 & 0.454 & RZ CMa & 9.162 & 0.448 & V510 Mon & 12.550 & 0.456 \\
AV Tau & 10.809 & 0.457 & RZ Gem & 9.973 & 0.454 & V Car & 7.915 & 0.447 \\
AX Aur & 11.955 & 0.461 & RZ Vel & 8.249 & 0.445 & V Vel & 7.888 & 0.448 \\
AX Vel & 8.120 & 0.448 & ST Tau & 8.897 & 0.452 & VX Pup & 8.718 & 0.449 \\
BE Mon & 9.609 & 0.452 & ST Vel & 8.158 & 0.442 & VY Car & 7.627 & 0.441 \\
BG Vel & 8.000 & 0.446 & SV Mon & 10.070 & 0.453 & VY Sgr & 5.862 & 0.453 \\
BK Aur & 10.207 & 0.453 & SW Vel & 8.457 & 0.433 & VZ Pup & 10.867 & 0.425 \\
BN Pup & 9.930 & 0.428 & SX Vel & 8.334 & 0.439 & WW Mon & 13.176 & 0.463 \\
BV Mon & 10.398 & 0.452 & SY Aur & 10.271 & 0.454 & WX Pup & 9.351 & 0.441 \\
CS Ori & 11.701 & 0.458 & T Vel & 8.084 & 0.448 & XX Mon & 11.854 & 0.451 \\
CV Mon & 9.362 & 0.452 & TW CMa & 9.788 & 0.445 & Y Aur & 9.692 & 0.453 \\
& & & TW Mon & 13.059 & 0.457 & YZ Aur & 11.668 & 0.459 \\
\hline
\end{tabular}

overtone (FO) pulsators have not been fundamentalized. Instead, different sets of P-W relations for either fundamental or FO pulsators have been calibrated.

We adopt here the individual distances estimated by Genovali et al. (in prep.) using these new P-W relations and single-epoch measurements or preferably mean magnitudes in the NIR bands. The NIR magnitudes used in Genovali et al. (in prep.) originate from four different data samples, ordered below according to their photometric accuracy and light curve coverage:

1. NIR photometry of 132 northern Cepheids from Monson \& Pierce (2011) with a complete coverage of the light curves;

2. data from the South African Astronomical Observatory previously published by Laney \& Caldwell (2007) and references therein, with a complete coverage of the light curves;

3. new, unpublished observations kindly provided by Laney (priv. comm.); the coverage of the light curves is currently only partial (6-12 points);

4. the 2MASS catalog, with only single-epoch observations. The mean magnitude is then estimated with a template light curve, the amplitude in the $V$-band and the epoch of maximum both available in the literature (Soszyński et al. 2005).

Three values of the distance modulus (and relative errors) have been computed in the three NIR bands, the conclusive value being their weighted mean. The results are in very good agreement with previous results from Monson \& Pierce (2011) and Storm et al. (2011a,b), in particular because they show no spurious trends with period or reddening. The reader interested in a complete description of the method adopted to estimate accurate distances together with a detailed analysis of the error budget is referred to Inno et al. (2013) and Genovali et al. (in prep.). However, it is worth mentioning that the error budget includes the errors affecting the Galactocentric distance of the Sun. Table 2 lists the Galactocentric distances for our high-resolution sample. Our whole sample (see Genovali et al., in prep.) is, to date, the largest homogeneous sample for accurate NIR distances of Galactic classical Cepheids.
We adopted $7.94 \mathrm{kpc}$ as the Galactocentric distance of the Sun, derived from type II Cepheids and RR Lyrae stars by Groenewegen et al. (2008). Fritz et al. (2011) obtained the same value from Red Clump stars in the Galactic center (GC) and an updated extinction curve toward the GC. A very similar value $(7.9 \mathrm{kpc})$ is derived by Reid et al. (2009) from the trigonometric parallax of Sgr B2, a massive star forming region very close to the GC. Similar values are reported by Matsunaga et al. (2013) from short-period variable stars close to the GC, by Trippe et al. (2008) from the statistical parallax of late-type stars in the GC (8.07 kpc), and by Majaess (2010) from OGLE RR Lyrae variables $(8.1 \mathrm{kpc})$. Also using RR Lyrae stars (statistical parallaxes), Dambis (2009) found a shorter distance of $7.58 \mathrm{kpc}$. On the other hand, Ghez et al. (2008) and Gillessen et al. (2009) derived a value of, respectively, 8.4 and $8.33 \mathrm{kpc}$ in monitoring stellar orbits around the massive black hole in the GC. These results are similar to those of Matsunaga et al. (2009) from Mira variables $(8.24 \mathrm{kpc})$. From a statistical analysis of the estimates published during the last 20 years, Malkin (2012) reports a final value of $7.98 \mathrm{kpc}$. Possible changes to the distance to the GC do not affect the conclusions of this investigation.

\subsection{Comparison with V-band-based distances}

Because of the recent advent of NIR detectors, the distances of Galactic Cepheids have been traditionally derived from $V$-band P-L relations $\left(\mathrm{PL}_{V}\right)$. Still, the amount of NIR data available for Galactic Cepheids is less than in the visible part of the spectrum, although the situation is continuously improving. Already in our previous papers (Lemasle et al. 2007, 2008; Pedicelli et al. 2009, 2010), we preferred to use NIR P-L relations: it is now widely accepted that the slopes and zero-points of the P-L relations are less dependent on the metallicity in the NIR than in the visible part of the spectrum. However, it is worth mentioning that no consensus has been reached yet on the amplitude of this so-called metallicity effect, neither from a theoretical nor from an empirical point of view (see, for instance, Fig. 1 in Romaniello et al. 2008). Moreover, the intrinsic dispersion of 
the P-L relations is significantly lower in the NIR than in the $V$-band, leading to lower systematic errors in the NIR.

Finally, another drawback of the P- $\mathrm{L}_{V}$-relations has been recently addressed. It is related to the metallicity dependence of the reddening correction: for a long time the main source of reddening values $E(B-V)$ has been the Fernie et al. (1995) database, where individual reddening values are computed from a periodcolor relation not taking into account the metallicity effect, although it is very likely that it has to be considered (Laney \& Stobie 1994; Bono et al. 1999). The first to adopt a revised version of the Fernie system were Tamman et al. (2003), and the works of Laney \& Caldwell (2007) and Fouqué et al. (2007) enabled us to correct any reddening value for the metallicity effect. Again, distances derived from NIR P-L relations are less affected by the possible remaining uncertainties in the determination of $E(B-V)$ because the total amount of reddening to be corrected is smaller in this spectral domain.

In this paper, we adopt distances derived from NIR P-W relations (Inno et al. 2013). This way, we benefit from the advantages of the P-W relations compared to their corresponding P-L relations (see Sect. 3.1) together with those of working in the NIR. As the relations have been derived for Magellanic Clouds Cepheids, we assume that the reddening law is the same in different systems. Figure 1a shows the overall good agreement between distances derived either from the $\mathrm{P}-\mathrm{L}_{V}$ taken from Luck et al. (2011), Luck \& Lambert (2011), or from the P-W $\mathrm{W}_{N I R}$ relations, because the difference is in general less than $5 \%$. Moreover, the difference is less than $2 \%$ for a significant part of our sample, especially close to the solar neighborhood, where one can note in Fig. $1 \mathrm{~b}$ the narrow distribution. On the other hand, the discrepancies are increasing when going further from the Sun, both toward the inner and toward the outer disk. Such a result is all but a surprise: the Cepheids located close to the Sun are not expected to be highly reddened, and therefore the discrepancies between $\mathrm{P}-\mathrm{L}_{V}$ and $\mathrm{P}-\mathrm{W}_{N I R}$ are expected to be minimal. When moving away from the Sun, one expects not only an increase of the reddening but also (from our previous knowledge of the Galactic metallicity gradient) an increase of the Cepheids' metallicity toward the inner disk and a decrease toward the outer disk. Both these characteristics will significantly affect the accuracy of the distances derived from $\mathrm{P}-\mathrm{L}_{V}$ relations. The values quoted above are in good agreement with uncertainties at a level of $13 \%$ reported by Luck \& Lambert (2011). Here we would like to stress that a discrepancy as small as 5\% at $10 \mathrm{kpc}$ translates into a significant discrepancy of $0.5 \mathrm{kpc}$. As the current sample of Cepheids with known abundances is largely located at $\pm 1-2 \mathrm{kpc}$ from the Sun, such an uncertainty on the distances does not yet strongly affect the determination of the gradients (differences $<0.005 \mathrm{dex} / \mathrm{kpc}$ ). However, this effect will certainly increase, together with an improved sampling (in terms of chemical composition) of the inner/outer disk or toward other Galactic quadrants, and affect the current conclusions.

\section{Results: abundances}

In this section we report the abundances obtained for the individual Cepheids and compare when possible with previous studies. For stars in common, we computed the mean value and standard deviation of the difference between our results and those of Luck \& Lambert (2011) and Luck et al. (2011) for S and Zr (we have only three stars in common with Yong et al. 2006). For a proper comparison, the results of Yong et al. (2006), Luck et al. (2011), and Luck \& Lambert (2011) have been scaled when needed to the solar chemical abundances of Grevesse et al. (1996). Values
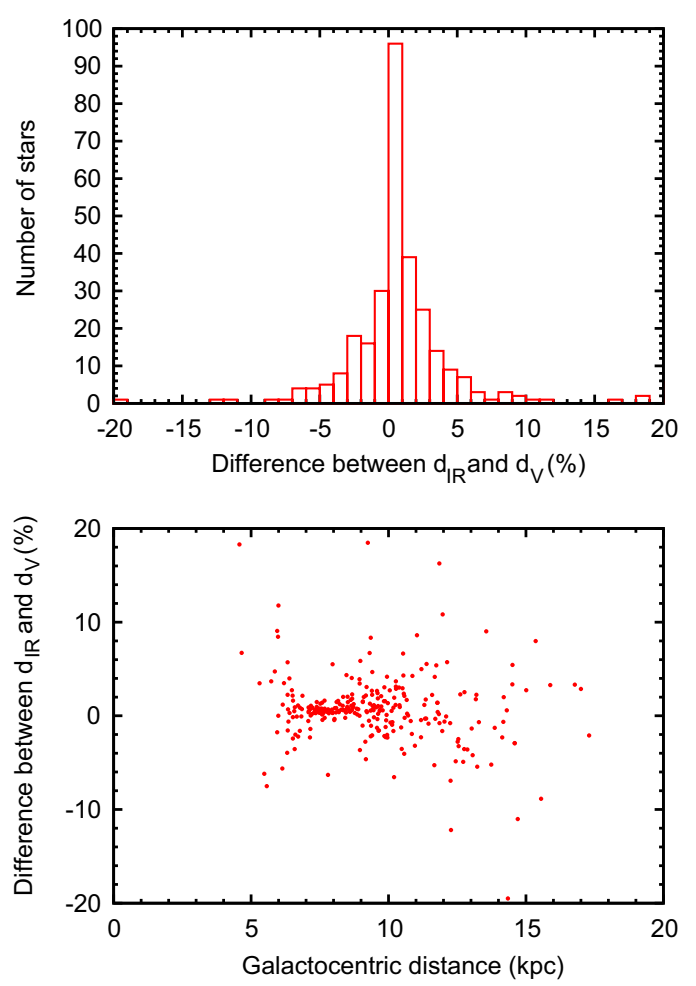

Fig. 1. a) Difference between distances derived from $\mathrm{P}-\mathrm{W}_{N I R}$ and $\mathrm{P}-\mathrm{L}_{V}$ relations as a function of the Galactocentric distance of the Cepheid. b) Comparison between distances derived from $\mathrm{P}-\mathrm{W}_{N I R}$ and $\mathrm{P}-\mathrm{L}_{V}$ relations.

are given in Table 3. As far as $[\mathrm{Fe} / \mathrm{H}]$ is concerned, we found an excellent agreement (differences $\leq 0.1$ dex in most cases) between our studies (Lemasle et al. 2007, 2008) and the results of Luck et al. (2006).

\subsection{Light elements}

Sodium: our sodium abundances cover a wide range of values, and, as expected for Galactic supergiants, the Cepheids in our sample are in most cases Na-overabundant. This overabundance is due to the synthesis of sodium via the $\mathrm{Ne}-\mathrm{Na}$ cycle in the convective cores of the intermediate-mass main sequence stars, which are the progenitors of Cepheids. After the first dredgeup, mixing brings the Na-enriched material into the radiative envelope of the stars (e.g., Sasselov 1986; Denissenkov 1994; Takeda et al. 2013, and references therein). Our results are in good agreement with Luck \& Lambert (2011).

Aluminium: Cepheids have aluminium abundances in the $[-0.3-+0.3 \mathrm{dex}]$ range. Once again, our results are in good agreement with Luck \& Lambert (2011). Although there are $6 \mathrm{Al}$ lines in our line list, they are usually weak in Cepheids and our results typically rely on 2-3 lines.

\section{2. $\alpha$ elements}

Magnesium: although magnesium abundances have been found mostly sub-solar in all of the previous Cepheid studies, Luck \& Lambert (2011) report higher $[\mathrm{Mg} / \mathrm{H}]$ values. The impact of the revision varies from star to star but as a whole can be considered as a shift upward of $\approx 0.2 \mathrm{dex}$. The method is exactly the same as in previous studies (including the value of $[\mathrm{Mg} / \mathrm{H}]_{\odot}$ ), and the possible systematics, which are examined thoroughly by Luck \& Lambert (2011), are extremely small. Moreover, Luck \& Lambert (2011) have several dozens of stars in common with 
Table 3. Mean value and standard deviation of the abundance difference between this study and Luck \& Lambert (2011), except for S and Zr, where the comparison is made with Luck et al. (2011).

\begin{tabular}{lcc}
\hline \hline Element & $\begin{array}{c}\text { Mean difference } \\
\text { dex }\end{array}$ & $\begin{array}{c}\sigma \\
\text { dex }\end{array}$ \\
\hline$[\mathrm{Na} / \mathrm{H}]$ & 0.12 & 0.11 \\
{$[\mathrm{Mg} / \mathrm{H}]$} & 0.19 & 0.15 \\
{$[\mathrm{Al} / \mathrm{H}]$} & 0.13 & 0.09 \\
{$[\mathrm{Si} / \mathrm{H}]$} & 0.11 & 0.07 \\
{$[\mathrm{~S} / \mathrm{H}]$} & 0.18 & 0.16 \\
{$[\mathrm{Ca} / \mathrm{H}]$} & 0.14 & 0.11 \\
{$[\mathrm{Y} / \mathrm{H}]$} & 0.16 & 0.11 \\
{$[\mathrm{Zr} / \mathrm{H}]$} & 0.17 & 0.13 \\
{$[\mathrm{La} / \mathrm{H}]$} & 0.20 & 0.14 \\
{$[\mathrm{Ce} / \mathrm{H}]$} & 0.14 & 0.11 \\
{$[\mathrm{Nd} / \mathrm{H}]$} & 0.20 & 0.12 \\
{$[\mathrm{Eu} / \mathrm{H}]$} & 0.18 & 0.16 \\
\hline
\end{tabular}

previous studies, so the shift of the $\mathrm{Mg}$ abundances cannot be related to azimuthal inhomogeneities in the Milky Way disk. Therefore it is hard to imagine another cause to explain the shift than the new line selection used by Luck \& Lambert (2011).

In our study we retained two $\mathrm{Mg}$ lines at $5711.09 \AA$ and 8736.02 A, respectively. However, the latter could not be observed in all of our spectra as the efficiency of the optical spectrographs (and in turn the $\mathrm{S} / \mathrm{N}$ ) decreases rapidly when approaching the NIR. We did not retain the $\mathrm{Mg}$ triplet around $6318 \AA$. These lines are close to an auto-ionization $\mathrm{Ca}$ line, which makes it harder to properly determine the continuum placement. This determination is crucial as these lines are generally weak in Cepheids. Because the $8736 \AA$ line is also a multiplet, it may also be better not to include it in our line list. On the other hand, $\mathrm{Mg}$ abundances derived from this line have always been consistent with those from the $5711 \AA$ line when both could be measured in the Cepheids of our sample.

Recently, Merle et al. (2011) studied the NLTE effects on some $\mathrm{Mg}$ and $\mathrm{Ca}$ lines in late-type giants/supergiants. For the $8736 \AA$ line, they found that the NLTE correction does not exceed -0.1 dex in the metallicity and gravity range of Galactic Cepheids. However, the temperature of their models does not exceed $5250 \mathrm{~K}$, while Galactic Cepheids have temperatures ranging from $4800 \mathrm{~K}$ to $6200 \mathrm{~K}$. As far as the $5711 \AA$ line is concerned and for the same temperature range, NLTE effects are negligible for Galactic Cepheids and are noticeable only below $[\mathrm{Fe} / \mathrm{H}]=-1.5$ dex.

Our results support the findings of Luck \& Lambert (2011): Cepheids in our sample have abundances ranging mostly from -0.3 dex to +0.3 dex, except toward the inner disk, where they reach higher values. They also fall on the same locus in the $[\mathrm{Mg} / \mathrm{H}]$ vs. $R_{\mathrm{G}}$ plane as the Cepheids in Luck \& Lambert (2011) and show a similar dispersion at a given Galactic radius (Fig. 2). The same applies to the Cepheids of Yong et al. (2006), for which we could compute NIR-based distances.

Silicium: our silicium abundances are in excellent agreement with those of Luck et al. (2011), Luck \& Lambert (2011), and previous studies. This is not surprising as a larger number of good Si lines (typically 10-12) can be measured in Cepheids.

Sulfur: our results show a greater scatter than for the other $\alpha$ elements, similar to what was found for sulfur in other studies (Luck et al. 2011). This is certainly due to the fact that our
$[\mathrm{S} / \mathrm{H}]$ abundances are based on typically $1-2$ relatively weak lines. In addition to this scatter, it seems that our sulfur abundances are shifted upward by $0.1-0.2$ dex, compared to those of Luck et al. (2011). As for $\mathrm{Mg}$, this could be due to the line selection because we include in our line list the lines at $6052.67 \AA$ and $6538.60 \AA$, which are not in the list of Luck et al. (2011). In contrast, however, they retained the lines at $7679.60 \AA$ and $7686.03 \AA$, which we did not measure. It is worth mentioning that we found no correlation between the $[\mathrm{S} / \mathrm{H}]$ scatter and the longitude.

It would be very important to reduce the scatter and to ascertain the sulfur abundances of Cepheids. Unlike C-N-O, sulfur is not processed in the intermediate and massive stars and thus traces the sulfur abundance in the interstellar medium at the time the Cepheid was born. This would enable a direct gradient comparison between Cepheids and the other tracers of the young population.

Calcium: although there are also many Ca lines in the Cepheid spectra, they are quite often strong lines and depart from a Gaussian profile, which makes them less suitable for abundance determination. At the end, we have typically 5-7 good Ca lines that have been properly measured. The dispersion is similar to the one obtained for Si. The agreement is also very good with previous studies. Among the lines in our list that have been studied by Merle et al. (2011), only the one at $6166.44 \AA$ suffers from a noticeable NLTE correction approaching 0.1 dex at $T_{\text {eff }}=5250 \mathrm{~K}$.

\subsection{Heavy elements}

Europium: our europium abundances are in good agreement with those from Luck \& Lambert (2011). With the exception of a few stars with rather large discrepancies, the differences are in general of the order of 0.15 dex or less. In both studies, $[\mathrm{Eu} / \mathrm{H}]$ is derived from only two lines, of which only one (at $6645.13 \AA$ ) is in common.

Other heavy elements ( $Y, \mathrm{Zr}, \mathrm{La}, \mathrm{Ce}, \mathrm{Nd})$ : we discuss the other heavy elements together as our comments will be very similar for all of them. When compared to Luck \& Lambert (2011), our measurements agree well for roughly half of our sample, which are mostly metal-rich Cepheids that are $\pm 2 \mathrm{kpc}$ from the Sun. The other half are in general more metal-poor Cepheids located beyond $\approx 10 \mathrm{kpc}$, and our heavy elements abundances are very often lower than those of Luck \& Lambert (2011). The discrepancies are largest in the case of Y, La, and $\mathrm{Nd}$, while Ce abundances agree very well. Because $\mathrm{Zr}$ has not been studied by Luck \& Lambert (2011), we compare with Luck et al. (2011), and our $\mathrm{Zr}$ abundances are in much better agreement than those of the other heavy elements.

The origin of this "bimodal" behavior is not clear. We have already mentioned that our approaches are very similar. Different causes can be invoked to understand this behavior:

- It could arise from systematics in the determination of the atmospheric parameters of the Cepheids. However, we found an excellent agreement for $[\mathrm{Fe} / \mathrm{H}]$ and most of the other elements we analyzed. Moreover, this could not explain why our heavy element abundances agree in general relatively well for the more metal-rich stars in our sample and not for the most metal-poor ones. We thus discard this possibility.

- It seems that we have to look in the direction of the absorption lines of the heavy elements. However, we can also exclude that the measurement errors (like a wrong fit of the 


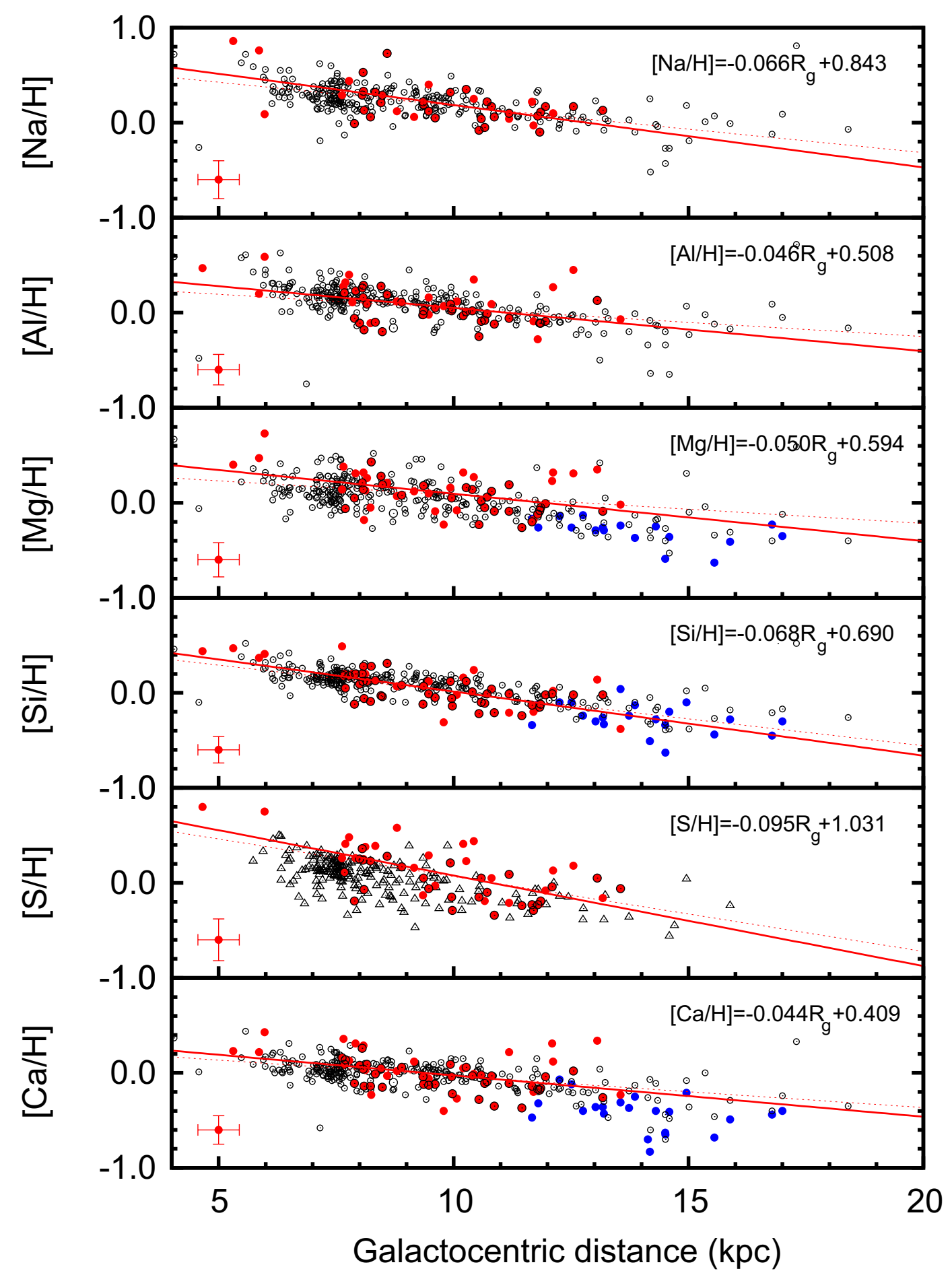

Fig. 2. Galactic abundance gradients from Cepheids for light and $\alpha$ elements. The full red dots are our data. We also show data from Luck \& Lambert (2011) (black open circles) and if not available, from Luck et al. (2011) (black open triangles). When stars are in common and the abundances fall within 0.2 dex, we overplotted black open circles (triangles) to the red dots. Yong et al. (2006) also provided data for several elements in the outer disk (full blue dots). All the distances are derived with a $\mathrm{PW}_{N I R}$ relation. A representative error bar is shown, including both the uncertainty on the atmospheric parameters and the rms uncertainty on the mean abundance $[\mathrm{X} / \mathrm{H}]$. We also indicate a linear fit to our data on the [4-15] kpc range (thick red line) and (for some elements) on the [7-15] kpc range (dashed red line).

lines or errors in the continuum placement) are larger for the lines of the heavy elements than in the case of other species. Moreover, it is easier to properly locate the continuum in the Cepheids that have sub-solar metallicities than in those that are more metal-rich than the Sun because the line blending decreases with metallicity. On the other hand, the more metal-poor stars in our sample are found toward the outer disk (a consequence of the abundance gradients) and therefore have lower $\mathrm{S} / \mathrm{N}$ due to their greater distances.

- We note that our analysis of heavy elements is based on a slightly larger number of lines than the other studies: we typically measure 4-6 Y lines, $1 \mathrm{Zr}$ line, 4-6 La lines, 2-3 Ce lines, and 2-4 Nd lines, while the line list of Kovtyukh \& Andrievsky (1999) lists only 2 lines for each 
B. Lemasle et al.: Galactic abundance gradients from Cepheids

Table 4. Slopes and zero-points of the abundance gradients for various elements.

\begin{tabular}{lccccccc}
\hline \hline Element & $\begin{array}{c}\text { Range } \\
\text { kpc }\end{array}$ & $\begin{array}{c}\text { Slope } \\
\text { dex/kpc }\end{array}$ & $\begin{array}{c}\text { Zero-point } \\
\text { dex }\end{array}$ & rms & $N$ & $\begin{array}{c}\text { Slope (previous studies) } \\
\text { dex/kpc }\end{array}$ & rms \\
\hline$[\mathrm{Na} / \mathrm{H}]$ & {$[4-15]$} & $-0.066 \pm 0.015$ & $0.843 \pm 0.146$ & 0.177 & 42 & $-0.047 \pm 0.003$ & 0.131 \\
& {$[7-15]$} & $-0.049 \pm 0.016$ & $0.673 \pm 0.161$ & 0.154 & 39 & & \\
{$[\mathrm{Mg} / \mathrm{H}]$} & {$[4-15]$} & $-0.050 \pm 0.013$ & $0.594 \pm 0.133$ & 0.185 & 54 & $-0.048 \pm 0.004$ & 0.160 \\
& {$[7-15]$} & $-0.030 \pm 0.015$ & $0.381 \pm 0.150$ & 0.174 & 51 & & \\
{$[\mathrm{Al} / \mathrm{H}]$} & {$[4-15]$} & $-0.046 \pm 0.013$ & $0.508 \pm 0.122$ & 0.170 & 55 & $-0.049 \pm 0.003$ & 0.130 \\
& {$[7-15]$} & $-0.030 \pm 0.014$ & $0.343 \pm 0.142$ & 0.164 & 52 & & \\
{$[\mathrm{Si} / \mathrm{H}]$} & {$[4-15]$} & $-0.068 \pm 0.009$ & $0.690 \pm 0.089$ & 0.139 & 62 & $-0.048 \pm 0.002$ & 0.088 \\
& {$[7-15]$} & $-0.057 \pm 0.011$ & $0.575 \pm 0.110$ & 0.140 & 58 & & \\
{$[\mathrm{~S} / \mathrm{H}]$} & {$[4-15]$} & $-0.095 \pm 0.015$ & $1.031 \pm 0.152$ & 0.206 & 52 & $-0.076 \pm 0.005$ & 0.159 \\
& {$[7-15]$} & $-0.079 \pm 0.017$ & $0.857 \pm 0.173$ & 0.202 & 50 & & \\
{$[\mathrm{Ca} / \mathrm{H}]$} & {$[4-15]$} & $-0.044 \pm 0.012$ & $0.409 \pm 0.119$ & 0.173 & 61 & $-0.041 \pm 0.003$ & 0.114 \\
& {$[7-15]$} & $-0.033 \pm 0.014$ & $0.302 \pm 0.138$ & 0.173 & 58 & & \\
{$[\mathrm{Y} / \mathrm{H}]$} & {$[4-15]$} & $-0.062 \pm 0.012$ & $0.569 \pm 0.114$ & 0.166 & 61 & $-0.061 \pm 0.003$ & 0.116 \\
{$[\mathrm{Zr} / \mathrm{H}]$} & {$[4-15]$} & $-0.078 \pm 0.016$ & $0.814 \pm 0.158$ & 0.162 & 41 & $-0.047 \pm 0.005$ & 0.127 \\
{$[\mathrm{La} / \mathrm{H}]$} & {$[4-15]$} & $-0.045 \pm 0.012$ & $0.541 \pm 0.111$ & 0.168 & 60 & $-0.031 \pm 0.004$ & 0.144 \\
{$[\mathrm{Ce} / \mathrm{H}]$} & {$[4-15]$} & $-0.043 \pm 0.012$ & $0.597 \pm 0.113$ & 0.158 & 55 & $-0.034 \pm 0.003$ & 0.133 \\
{$[\mathrm{Nd} / \mathrm{H}]$} & {$[4-15]$} & $-0.046 \pm 0.013$ & $0.593 \pm 0.131$ & 0.165 & 55 & $-0.037 \pm 0.003$ & 0.114 \\
{$[\mathrm{Eu} / \mathrm{H}]$} & {$[4-15]$} & $-0.066 \pm 0.013$ & $0.778 \pm 0.122$ & 0.184 & 59 & $-0.042 \pm 0.005$ & 0.171 \\
& {$[7-15]$} & $-0.056 \pm 0.015$ & $0.671 \pm 0.153$ & 0.184 & 55 & & \\
\hline
\end{tabular}

Notes. We also indicate the root mean square (rms) of the residuals and the number of data points for the fit. For some elements, we also computed the gradient over a different distance range. The gradient values and rms obtained by Luck \& Lambert (2011) are tabulated for comparison (Luck et al. (2011) for $\mathrm{S}$ and $\mathrm{Zr}$ ).

heavy element. For the lines that are in common, we checked that we have similar atomic parameters, except for the $\mathrm{Y}$ line at $6795.41 \AA$. For this $\mathrm{Y}$ line we used a $\log g f$ value of -1.03, while Kovtyukh \& Andrievsky (1999) used a value of -1.20 . Yong et al. (2006) measured one Eu line and four La lines, among which three are in common with our own line list and for which they retained the same atomic parameters. We also note that the differences are greater for the elements where we used a larger number of lines than Luck \& Lambert (2011) (Y, La, Nd), while the agreement is quite good when we used a similar number of lines $(\mathrm{Zr}, \mathrm{Ce}, \mathrm{Eu})$.

- As the atomic parameters for the lines in common are identical or very similar in most cases, it seems unlikely that they account for much of the difference. It is probable that at least some of the lines of these elements (which are, moreover, found in their ionized state) are altered by NLTE effects. Selecting different lines (that are not affected in the same way by NLTE effects) in different line lists could then lead to the discrepant results we observe. None of the studies compared here (Yong et al. 2006; Luck et al. 2011; Luck \& Lambert 2011; this study) computed NLTE effects for the heavy elements or took into account hyperfine structure corrections. Repeating with other elements the NLTE analysis of barium in Milky Way Cepheids made by Andrievsky et al. (2013) would certainly help to clarify the situation.

\section{Results: gradients}

When examining the abundance trends with Galactocentric distance, we first considered simple, linear gradients. Figure 2 displays linear gradients for several light $(\mathrm{Na}, \mathrm{Al})$ and $\alpha$ elements, while Fig. 3 shows the gradients for the heavy elements. Details about the linear regression are given in Table 4.

Because $\mathrm{Na}$ and possibly $\mathrm{Al}$ are modified prior to the Cepheid evolutionary stage, their gradients should only be considered as indicative. For $\mathrm{Na}$, the high value obtained for the slope of the gradient $(-0.066 \mathrm{dex} / \mathrm{kpc})$ is largely due to the influence of a couple of Na-rich stars in the inner disk. If in contrast we compute the gradient only in the range [7-15] kpc, the value we obtain $(-0.049 \mathrm{dex} / \mathrm{kpc})$ is very similar to the results of Luck et al. (2011) and Luck \& Lambert (2011), which are $-0.044 \mathrm{dex} / \mathrm{kpc}$ and $-0.047 \mathrm{dex} / \mathrm{kpc}$, respectively. Our results are also very similar for the $\mathrm{Al}$ gradient: we found a slope of $-0.046 \mathrm{dex} / \mathrm{kpc}$ over the whole Galactic disk, while Luck et al. (2011), Luck \& Lambert (2011) gave slopes of -0.053 and $-0.49 \mathrm{dex} / \mathrm{kpc}$, respectively. This value is also similar to the slope of $-0.048 \mathrm{dex} / \mathrm{kpc}$ found by Daflon \& Cunha (2004b) from OB stars.

For the $\alpha$ elements, Fig. 2 shows that our abundance trends with Galactocentric distance agree very well with the studies of Luck et al. (2011), Luck \& Lambert (2011), and Yong et al. (2006). Indeed, the locus of the stars in the $[\mathrm{M} / \mathrm{H}]$ vs. $R_{\mathrm{G}}$ plane and the dispersion around the slope match very well. The actual value of the slope (tabulated in Table 4) might be affected in some cases by the 3-4 $\alpha$ rich stars in the inner disk and/or by 2-4 stars at 12-14 kpc with higher $\alpha$-abundances than the other Cepheids at these Galactocentric distances. In particular for $\mathrm{Mg}$, the best fit seems to be artificially shifted by $\approx+0.2$ dex from the rest of the sample under the combined influence of these stars. From the figure it seems, however, that the slope is correct, and indeed our computed slope $(-0.050 \mathrm{dex} / \mathrm{kpc})$ is in excellent agreement with the results of Luck et al. (2011), and Luck \& Lambert (2011), who both found a slope of $-0.048 \mathrm{dex} / \mathrm{kpc}$, and with those of Daflon \& Cunha (2004b), who found a slope of $-0.052 \mathrm{dex} / \mathrm{kpc}$. We already mentioned that our $[\mathrm{S} / \mathrm{H}]$ abundances are higher by $+0.1-+0.2$ dex than those of Luck et al. (2011), which is confirmed by Fig. 2. We find, however, a similar slope $(-0.079 \mathrm{dex} / \mathrm{kpc}$ vs. $-0.076 \mathrm{dex} / \mathrm{kpc})$ if we ignore the two inner disk Cepheids, while Daflon \& Cunha (2004b) report a much shallower slope $(-0.040 \mathrm{dex} / \mathrm{kpc})$. The dispersion is much lower for $\mathrm{Si}$ and, to a slightly lesser extent, for $\mathrm{Ca}$ because their abundances are determined with a larger number of lines. Given the size of the respective error bars, this dispersion is certainly closer to reflecting a real star-to-star 


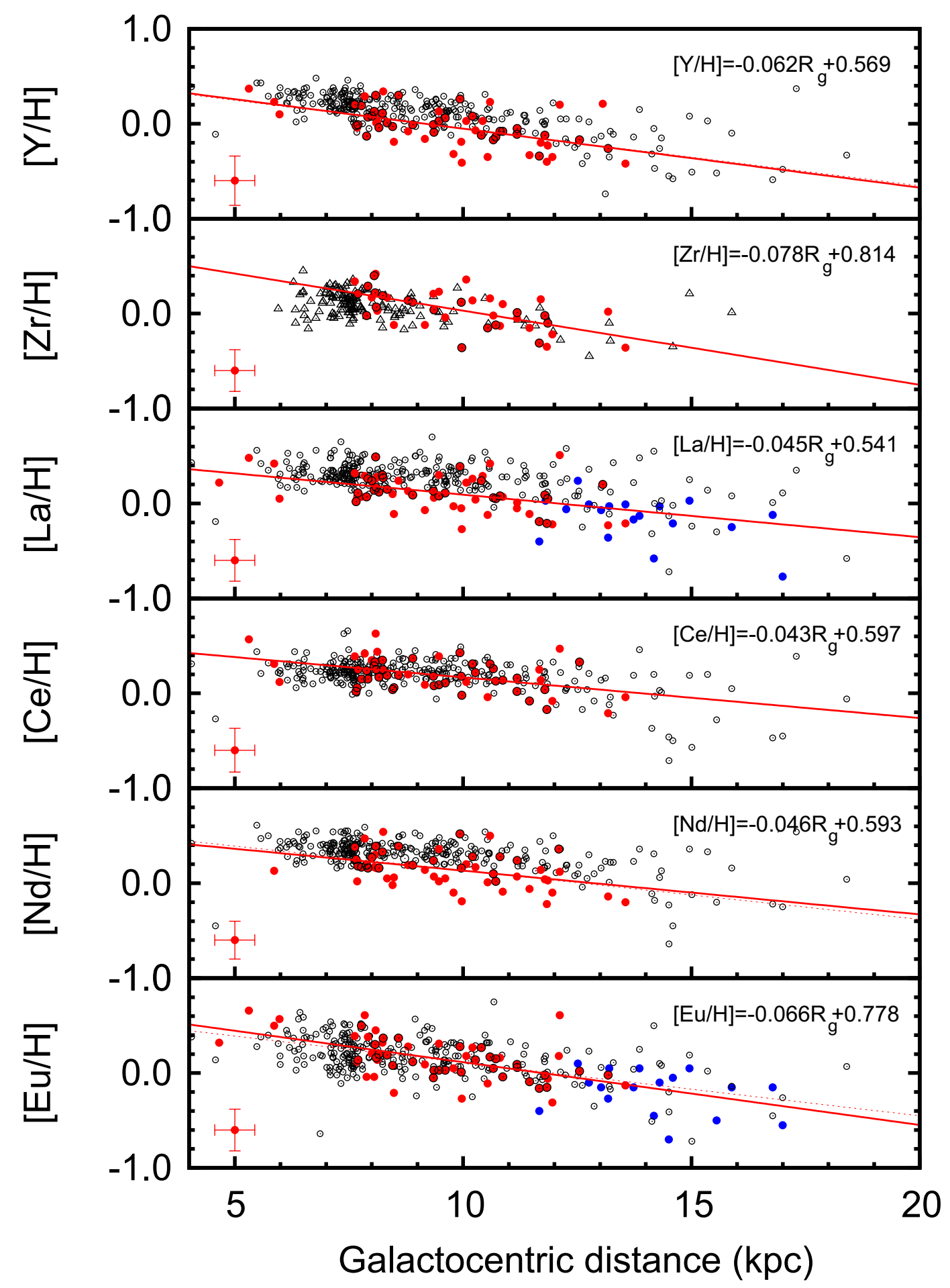

Fig. 3. Same as Fig. 2, but for heavy elements.

scatter, while it is probably dominated by the uncertainties of the abundance determinations for the other elements. Our Ca slope $(-0.044 \mathrm{dex} / \mathrm{kpc})$ is very close to those determined by Luck et al. (2011) and Luck \& Lambert (2011), which are $-0.040 \mathrm{dex} / \mathrm{kpc}$ and $-0.041 \mathrm{dex} / \mathrm{kpc}$, respectively. It is surprising to note that the inclusion (or not) of the four slightly more Si-rich stars in the inner disk noticeably changes the value of the slope, from $-0.068 \mathrm{dex} / \mathrm{kpc}$ to $-0.057 \mathrm{dex} / \mathrm{kpc}$. Both these values are larger than those reported by Luck et al. (2011) and Luck \& Lambert (2011) (-0.049 dex/kpc and $-0.048 \mathrm{dex} / \mathrm{kpc}$, respectively), and the difference is even larger with Daflon \& Cunha (2004b), who found a slope of only $-0.040 \mathrm{dex} / \mathrm{kpc}$. The lower value for the slope has our preference as it better fits the data of Luck et al. (2011) and Luck \& Lambert (2011), while the abundances of the inner disk Cepheids do not deviate much from this slope. On the other hand, the higher values better fits the data of Yong et al. (2006).

The slope values are also in good $(\mathrm{Mg}, \mathrm{Si})$ or very good (Ca) agreement with those derived by Cescutti et al. (2007) in their chemical evolution models, which are $-0.039,-0.045$, $-0.047 \mathrm{dex} / \mathrm{kpc}$ for $\mathrm{Mg}, \mathrm{Si}$, and $\mathrm{Ca}$, respectively. However, they report for sulfur a similar slope $(-0.047 \mathrm{dex} / \mathrm{kpc})$ that is much 
shallower than the ones found in this study or the study by Luck et al. (2011). The steeper slope found for sulfur should then be regarded with some caution, and the determination of the sulfur abundances in Cepheids clearly deserves further investigation.

Cescutti et al. (2007) also report that the $\alpha$ element abundances predicted by their model around the solar circle are slightly higher than the ones reported at that time from Cepheid observation, and that the difference is much greater for $\mathrm{Mg}$. In this respect, the upward revision of the $\mathrm{Mg}$ abundances by Luck \& Lambert (2011), supported here, improves the agreement between the model and the observations. From a different point of view, Piovan et al. (2011) constructed a model with radial flows of dust and gas but no radial migration of stars. The model reproduces well the observations of gradients from different tracers, in particular the Cepheids, for a good number of proton-capture and $\alpha$ elements.

The existence of radial abundance gradients for the heavy elements has been more controversial than for the other elements. Their spectral lines are not very numerous, either rather weak or too strong (especially in the case of $\mathrm{Ba}$ ), and quite often located in crowded parts of the spectra, which involves blending. These difficulties lead to an increased observational scatter and hence to inconclusive results in terms of gradients. It is only in the recent studies of Luck et al. (2011) and Luck \& Lambert (2011) that the existence of gradients has been recognized for all of the heavy elements measured. On the other hand, Andrievsky et al. (2013) found no gradient at all in a recent NLTE study of barium in Cepheids over the whole Galactic disk.

Our results confirm the existence of radial abundance gradients (within the context of an LTE analysis) for all the heavy elements we studied (Y, Zr, La, Ce, Nd, Eu). For Ce, the locus of our points and the value of the slope are in good agreement with Luck \& Lambert (2011), while for $\mathrm{Zr}$ the slope is overestimated (despite a similar locus) when compared to Luck et al. (2011), probably due to the absence of $\mathrm{Zr}$ measurements below $7.5 \mathrm{kpc}$ in our Cepheid sample. The europium trend with Galactocentric distance also seems to be in very good agreement when comparing our results to the observations of Luck \& Lambert (2011) and Yong et al. (2006) in Fig. 3. It is then surprising that the slopes are not in good agreement $(-0.066$ vs. $-0.042 \mathrm{dex} / \mathrm{kpc})$. Again, this is probably linked to the large number of stars with a solar-like abundance around $7 \mathrm{kpc}$ in the sample of Luck \& Lambert (2011), which we do not have in our sample, together with the influence of the Eu-rich inner disk Cepheids. Indeed, when the latter are not taken into account, the slope goes down to $-0.056 \mathrm{dex} / \mathrm{kpc}$. As far as the other elements are concerned, the slopes are in good agreement $(\mathrm{Y}, \mathrm{Nd})$ with previous results, despite the differences in abundance results discussed in Sect. 4. The discrepancies are largest in the case of La, and for this element our gradients match much better to the outer disk Cepheids of Yong et al. (2006) than to the large sample of Luck \& Lambert (2011). We believe that this effect is related to the La absorption lines that are included or not in the line lists used by the different authors. The slope we obtain $(-0.045 \mathrm{dex} / \mathrm{kpc})$ is then much steeper than the $-0.031 \mathrm{dex} / \mathrm{kpc}$ reported by Luck et al. (2011). Cescutti et al. (2007) noted that the La abundances in the solar neighborhood of Luck et al. (2006) were higher than those predicted by their model. As a result, the slope predicted by the model $(-0.032 \mathrm{dex} / \mathrm{kpc}$ in the [4-14] kpc range) was steeper than the one seen in the data available at that time. It is, however, close to the current value proposed by Luck \& Lambert (2011). Considering only the objects located within $13 \mathrm{kpc}$ from the Galactic center, Yong et al. (2012) compared the slope of the gradients for the Cepheids and for the young $(<1 \mathrm{Gyr})$ open clusters in their compilation (see their Fig. 32). The slopes are in very close agreement for all of the elements except La, where the difference reaches $\approx 0.05$ dex (the open clusters gradient has a larger value than the Cepheid gradient). It should be noted that the uncertainties on the La gradient from open clusters are also much greater than for any other element. Clearly La deserves further investigation, both from a Cepheid and from an open cluster point of view. While $\mathrm{Y}$ and $\mathrm{Nd}$ show a similar behavior to La, the differences are less pronounced and the slopes of our gradients are in good agreement with Luck \& Lambert (2011).

A steeper slope for the iron gradient in Cepheids toward the inner disk was first reported by Andrievsky et al. (2002b) and supported by Pedicelli et al. $(2009,2010)$ and more recently on a larger sample by Genovali et al. (2013). On the basis of the current (small) sample of Cepheids toward the inner disk, it is impossible to say if such a distinctive feature also holds for the other elements: the shallower slopes found when the inner disk Cepheids are discarded seem to speak in favor of this scenario; on the other hand this could simply be caused by the severe undersampling of inner disk Cepheids. We plan to focus on this region in more detail in a forthcoming paper (Genovali et al., in prep.).

In contrast with previous studies, we found no clear evidence of a flattening of the gradient in the outer disk, as was already pointed out by Luck et al. (2011) and Luck \& Lambert (2011). This is in contrast to the young population in distant galaxies and to the open clusters in the Milky Way. Jacobson et al. (2011b), Yong et al. (2012), and most of the previous open clusters studies report a flattening of the gradient in the outer disk. When splitting the sample in three age bins, Jacobson et al. (2011b) report a possible flattening of the gradient, but also a possible change in the location of the transition zone with time. The outer disk flattening seems to occur around $10 \mathrm{kpc}$ for the oldest clusters, while $[\mathrm{Fe} / \mathrm{H}]$ in the intermediate-age clusters decreases linearly and reaches the same ceiling value only around $14 \mathrm{kpc}$ (where the distribution of the intermediate-age clusters stops). It is impossible to draw any firm conclusion yet due the unequal sampling of each bin (each age bin contains a different number of clusters and the sampling in terms of Galactocentric distance is also different). Because the recent studies could not detect a flattening of the Cepheid gradient in the outer disk, the Cepheids would then easily fit in that context. A comprehensive comparison between Cepheids and open clusters has been made by Yong et al. (2012); we will not repeat the exercise here. The simulations of Minchev et al. (2013) also show no flat gradient in the outer disk for the young stars. They found a strong flattening of the gradient for the old stars, which was also found by Wang $\&$ Zhao (2013), but the young populations are much less affected. As a result, the initial and the present-day gradient are very similar out to $\approx 12 \mathrm{kpc}$, where some flattening occurs. Indeed, the differences between the initial/final slopes are very small and anyway smaller than the uncertainties on the observed gradient.

Large surveys combining photometry and spectroscopy enable us to derive the radial velocities and the atmospheric parameters of numerous stars. The size of the samples and their high internal consistency compensates for the somewhat less accurate abundances and distances compared to the tracers commonly used to study the abundance gradients in the Milky Way. From the Geneva-Copenhagen Survey, Nordström et al. (2004) derived a value of $-0.076 \pm 0.014 \mathrm{dex} / \mathrm{kpc}$ for stars younger than $1.5 \mathrm{Gyr}$ in the solar neighborhood $(\approx[7-9] \mathrm{kpc})$. From the third data release (DR3) of the RAVE survey (Steinmetz et al. 2006), Coşkunoğlu et al. (2012) derived for young, F-type stars a radial metallicity $[\mathrm{M} / \mathrm{H}]$ gradient of $-0.051 \pm 0.005 \mathrm{dex} / \mathrm{kpc}$ 
similar to the Cepheid gradient. Again from the RAVE DR3 data but this time for red clump stars in the thin disk, Bilir et al. (2012) report a slightly shallower gradient of $[\mathrm{M} / \mathrm{H}]=-0.041 \pm 0.005 \mathrm{dex} / \mathrm{kpc}$. From MSTO stars (which are older than Cepheids) in the SEGUE data (Yanni et al. 2009), Cheng et al. (2012a) found a gradient of $-0.066 \mathrm{dex} / \mathrm{kpc}$ for stars at $0.15<|z|<0.25 \mathrm{kpc}$, with a gradient flattening toward higher altitudes. This value is close to the one obtained for Cepheids. However, it should be noted that most of the Cepheids are located below $|z|=0.15 \mathrm{kpc}$. Moreover, the SEGUE subsample considered contains several hundreds of stars in the [0.15-0.25] kpc layer, but over a range of Galactocentric distances limited to the [7-10] kpc region. Taking advantage of a large spectroscopic sample collected in preparation for the CoRoT mission (Loeillet et al. 2008), Gazzano et al. (2013) determined the atmospheric parameters of numerous Milky Way disk stars with the help of the MATISSE algorithm (Recio-Blanco et al. 2006). They report a radial metallicity gradient of $-0.097 \pm 0.015 \mathrm{dex} / \mathrm{kpc}$ with giant stars and $-0.053 \pm 0.015 \mathrm{dex} / \mathrm{kpc}$ with dwarfs; the latter in very good agreement with the value obtained from Cepheids. Most of the results quoted above concern the iron gradient and sometimes also the $\alpha$ elements (Cheng et al. 2012b; Gazzano et al. 2013). Possible comparisons for a larger number of elements await the results of the ongoing large spectroscopic surveys like APOGEE (Majewski et al. 2007), the GAIA-ESO survey (Gilmore et al. 2012), or HERMES (Freeman 2012).

\section{Conclusions}

We used high-resolution spectra (FEROS, ESPADONS, NARVAL) to determine the abundances of several light ( $\mathrm{Na}$, $\mathrm{Al}), \alpha(\mathrm{Mg}, \mathrm{Si}, \mathrm{S}, \mathrm{Ca}$ ), and heavy elements (Y, Zr, La, Ce, Nd, $\mathrm{Eu})$ in a sample of 65 Milky Way Cepheids. Combining these results with accurate distances from $\mathrm{p}-\mathrm{W}$ relations in the NIR enables us to study the abundance gradients in the Milky Way. Our results are in good agreement with previous studies from Cepheids or other tracers. In particular:

- We confirm an upward shift of $\approx 0.2$ dex for the Mg abundances first reported by Luck \& Lambert (2011).

- The locus of our points is for most of the elements in good agreement with previous Cepheid studies (Yong et al. 2006; Luck et al. 2011; Luck \& Lambert 2011). The slopes are sometimes discrepant, mainly due to sampling effects, as a few points sometimes have a strong influence on the determination of the slope. Our sample, originally designed to study either the outer disk or the very inner disk, lacks targets in the solar neighborhood.

- We confirm the existence of a gradient for all the heavy elements in the context of an LTE analysis. Moreover, for Y, Nd, and especially La, we find lower abundances for Cepheids in the outer disk than reported in previous studies, leading to steeper slopes for the gradients. We attribute this effect to the differences in the line lists used by different groups. On the other hand, Andrievsky et al. (2013) report no gradient for an NLTE analysis of Ba. Further investigations are needed to assess the behavior of the heavy elements and quantify, in particular, the role of the asymptotic giant branch stars in the chemical evolution of the Milky Way.

- Current data do not support a flattening of the gradients in the outer disk, in agreement with the observations of Luck et al. (2011), Luck \& Lambert (2011), and the simulations of
Minchev et al. (2013). This is different from what is observed in open cluster, but remains coherent with a picture where the transition zone between the inner disk and the outer disk would move outward with time (Jacobson et al. 2011b).

Acknowledgements. We thank the anonymous referee for the careful reading of our manuscript and the valuable comments. This work was partially supported by PRIN-INAF 2011 "Tracing the formation and evolution of the Galactic halo with VST" (PI: Marconi) and by PRIN-MIUR (2010LY5N2T) "Chemical and dynamical evolution of the Milky Way and Local Group galaxies" (PI: Matteucci). G.B. thanks ESO for support as science visitors. V.K. is grateful for the support from the Swiss National Science Foundation, project SCOPES No. IZ73Z0-128180. B.L. thanks the French Programme National de Physique Stellaire (PNPS) for the financial support of the observing missions.

\section{References}

Afflerbach, A., Churchwell, E., Acord, J. M., et al. 1996, ApJS, 106, 423 Afflerbach, A., Churchwell, E., \& Werner, M. W. 1997, ApJ, 478, 190 Andrievsky, S. M., Kovtyukh, V. V., Luck, R. E., et al. 2002a, A\&A, 381, 32 Andrievsky, S. M., Bersier, D., Kovtyukh, V. V., et al. 2002b, A\&A, 384, 140 Andrievsky, S. M., Kovtyukh, V. V., Luck, R. E., et al. 2002c, A\&A, 392, 491 Andrievsky, S. M., Luck, R. E., Martin, P., \& Lépine, J. R. D. 2004, A\&A, 413, 159

Andrievsky, S. M., Luck, R. E., \& Kovtyukh, V. V. 2005, AJ, 130, 1880

Andrievsky, S. M., Lépine, J. R. D., Korotin, S. A., et al. 2013, MNRAS, 428, 3252

Balser, D. S., Rood, R. T., Bania, T. M., \& Anderson, L. 2011, ApJ, 738, 27

Barker, M. K., Sarajedini, A., Geisler, D., Harding, P., \& Schommer, R. 2007, AJ, 133, 1138

Bilir, S., Karaali, S., Ak, S., et al. 2012, MNRAS, 421, 3362

Binney, J., \& Tremaine, S. 2008, Galactic Dynamics, 2nd edn. (Princeton University Press)

Bird, J. C., Kazantzidis, S., \& Weinberg, D. H. 2012, MNRAS, 420, 913

Bird, J. C., Kazantzidis, S., Weinberg, D. H., et al. 2013, ApJ, 773, 43

Bono, G., Caputo, F., Castellani, V., \& Marconi, M. 1999, ApJ, 512, 711

Bono, G., Marconi, M., Cassisi, S., et al. 2005, ApJ, 621, 966

Bono, G., Caputo, F., Fiorentino, G., Marconi, M., \& Musella, I. 2008, ApJ, 684, 102

Bono, G., Caputo, F., Marconi, M., \& Musella, I. 2010, ApJ, 715, 277

Bragaglia, A., Sestito, P., Villanova, S., et al. 2008, A\&A, 480, 79

Bresolin, F., Ryan-Weber, E., Kennicutt, R. C., \& Goddard, Q. 2009, ApJ, 695, 580

Bresolin, F., Gieren, W., Kudritzki, R.-P., et al. 2009, ApJ, 700, 309

Carraro, G., Geisler, D., Villanova, S., Frinchaboy, P. M., \& Majewski, S. R. 2007, A\&A, 476, 217

Carretta, E., Bragaglia, A., Gratton, R. G., \& Tosi, M. 2004, A\&A, 422, 951

Carretta, E., Bragaglia, A., Gratton, R. G., \& Tosi, M. 2005, A\&A, 441, 131

Carretta, E., Bragaglia, A., \& Gratton, R. G. 2007, A\&A, 473, 129

Cescutti, G., Matteucci, F., François, P., \& Chiappini, C. 2007, A\&A, 462, 943

Charbonneau, P. 1995, ApJSS, 101, 309

Chen, L., Hou, J. L., \& Wang, J. J. 2003, AJ, 125, 1397

Cheng, J. Y., Rockosi, C. M., Morrison, H. L., et al. 2012a, ApJ, 746, 149

Cheng, J. Y., Rockosi, C. M., Morrison, H. L., et al. 2012b, ApJ, 752, 51

Chiappini, C., Matteucci, F., \& Gratton, R. 1997, ApJ, 477, 765

Chiappini, C., Matteucci, F., \& Romano, D. 2001, ApJ, 554, 1044

Colavitti, E., Cescutti, G., Matteucci, F., \& Chiappini, C. 2008, A\&A, 496, 429

Coskunoğlu, B., Ak, S., Bilir, S., et al. 2012, MNRAS, 419, 2844

Costa, R. D. D., Uchida, M. M. M., \& Maciel, W. J. 2004, A\&A, 423, 199

Daflon, S., \& Cunha, K. 2004, ApJ, 617, 1115

Dambis, A. K. 2009, MNRAS, 396, 553

Deharveng, L., Pena, M., Caplan, J., \& Costero, R. 2000, MNRAS, 311, 329

Denissenkov, P. A. 1994, A\&A, 287, 113

Di Matteo, P., Haywood, M., Combes, F., Semelin, B., \& Snaith, O. N. 2013, A\&A, 553, A102

Edva Steinmetz, M., Zwitter, T., Siebert, A., et al. 1993, A\&A, 275, 101

Esteban, C., García-Rojas, J., Peimbert, M., et al. 2005, ApJ, 618, 95

Fernie, J. D., Beattie, B., Evans, N. R., \& Seager, S. 1995, IBVS, 4148

Few, C. G., Courty, S., Gibson, B. K., et al. 2012, MNRAS, 424, 11

Fiorentino, G., Marconi, M., Musella, I., \& Caputo, F. 2007, A\&A, 476, 863

Fouqué, P., Arriagada, P., Storm, J., et al. 2007, A\&A, 476, 73

François, P., \& Matteucci, F. 1993, A\&A, 280, 136

Freeman, K. C. 2012, ASPC, 458, 393

Friel, E. D., Janes, K. A., Tavarez, M., et al. 2002, AJ, 124, 2693

Friel, E. D., Jacobson, H. R., \& Pilachowski, C. A. 2010, AJ, 139, 1942

Fritz, T. K., Gillessen, S., Dodds-Eden, K., et al. 2011, ApJ, 737, 73 
Fu, J., Hou, J. L., Yin, J., \& Chang, R. X. 2009, ApJ, 696, 668

Fu, J., Kauffmann, G., Huang, M., et al. 2013, MNRAS, in press [arXiv: 1303. 5586]

Gazzano, J.-C., Kordopatis, G., Deleuil, M., et al. 2013, A\&A, 550, A125

Genovali, K., Lemasle, B., Bono, G., et al. 2013, A\&A, 554, A132

Gerke, J. R., Kochanek, C. S., Prieto, J. L., Stanek, K. Z., \& Macri, L. M. 2011, ApJ, 743, 176

Ghez, A. M., Salim, S., Weinberg, N. N., et al. 2008, ApJ, 689, 1044 Gillessen, S., Eisenhauer, F., Trippe, S., et al. 2009, ApJ, 692, 1075

Gilmore, G., Randich, S., Asplund, M., et al. 2012, The Messenger, 147, 25

Grevesse, N., Noels, A., \& Sauval, J. 1996, ASPC, 99, 117

Groenewegen, M. A. T., Udalski, A., \& Bono, G. 2008, A\&A, 481, 441

Gummersbach, C. A., Kaufer, D. R., Schäfer, D. R., Szeifert,T., \& Wolf, B. 1998, A\&A, 338, 881

Harris, H. C. 1981, AJ, 86, 707

Harris, H. C. 1984, ApJ, 282, 655

Henry, R. B. C., Kwitter, K. B., \& Balick, B. 2004, AJ, 127, 2284

Henry, R. B. C., Kwitter, K. B., Jaskot, A. E., et al. 2010, ApJ, 724, 748

Inno, L., Matsunaga, N., Bono, G., et al. 2013, ApJ, 764, 84

Jacobson, H. R., Friel, E. D., \& Pilachowski, C. A. 2008, AJ, 135, 2341

Jacobson, H. R., Friel, E. D., \& Pilachowski, C. A. 2009, AJ, 137, 4753

Jacobson, H. R., Friel, E. D., \& Pilachowski, C. A. 2011a, AJ, 141, 58

Jacobson, H. R., Friel, E. D., \& Pilachowski, C. A. 2011b, AJ, 142, 59

Kobayashi, C., \& Nakasato, N. 2011, ApJ, 729, 16

Kovtyukh, V. V. 2007, MNRAS, 378, 617

Kovtyukh, V. V., \& Andrievsky, S. M. 1999, A\&A, 351, 597

Kovtyukh, V. V., \& Gorlova, N. I. 2000, A\&A, 358, 587

Kovtyukh, V. V., Andrievsky, S. M., Belik, S. I., \& Luck, R. E. 2005a, AJ, 129, 433

Kovtyukh, V. V., Wallerstein, G., \& Andrievsky, S. M. 2005b, PASP, 117, 1173

Kudritzki, R.-P., Urbaneja, M. A., Bresolin, F., et al. 2008, ApJ, 681, 269

Kupka, F., Piskunov, N. E., Ryabchikova, T. A., Stempels, H. C., \& Weiss, W. W. 1999, A\&AS, 138, 119

Lacey, C. G., \& Fall, S. M. 1985, ApJ, 290, 154

Laney, C. D., \& Caldwell, J. A. R. 2007, MNRAS, 377, 147

Laney, C. D., \& Stobie, R. S. 1994, MNRAS, 266, 441

Lemasle, B., François, P., Bono, G., et al. 2007, A\&A, 467, 283

Lemasle, B., François, P., Piersimoni, A., et al. 2008, A\&A, 490, 613

Loeillet, B., Bouchy, F., Deleuil, M., et al. 2008, A\&A, 479, 865

Luck, R. E., \& Andrievsky, S. M. 2004, AJ, 128, 343

Luck, R. E., \& Lambert, D. L. 2011, AJ, 142, 136

Luck, R. E., Gieren, W. P., Andrievsky, S. M., et al. 2003, A\&A, 401, 939

Luck, R. E., Kovtyukh, V. V., \& Andrievsky, S. M. 2006, AJ, 132, 902

Luck, R. E., Andrievsky, S. M., Fokin, A., \& Kovtyukh, V. V. 2008, AJ, 136, 98

Luck, R. E., Andrievsky, S. M., Kovtyukh, V. V., Gieren, W., \& Graczyk, D. 2011, AJ, 142, 51

Maciel, W. J., \& Costa, R. D. D. 2009, in IAU Symp. 254, eds. J. Andersen, J. Bland-Hawthorn, \& B. Nordström, 38

Maciel, W. J., \& Quireza, C. 1999, A\&A, 345, 629

Maciel, W. J., Costa, R. D. D., \& Uchida, M. M. M. 2003, A\&A, 397, 667

Madore, B. F. 1982, ApJ, 253, 575

Madore, B. F., \& Freedman, W. F. 2009, ApJ, 696, 1498

Magrini, L., Sestito, P., Randich, S., \& Galli, D. 2009a, A\&A, 494, 95

Magrini, L., Stanghellini, L., \& Villaver, E. 2009b, ApJ, 696, 729

Magrini, L., Randich, S., Zoccali, M., et al. 2010, A\&A, 523, A11

Majaess, D. 2010, Acta Astron., 60, 55

Majaess, D., Turner, D., \& Gieren, W. 2011, ApJ, 741, 36

Majewski, S. R., Skrutskie, M. F., Schiavon, R. P., et al. 2007, BAAS, 39, 962

Malkin, Z. 2012, unpublished [arXiv: 1202.6128]

Matsunaga, N., Kawadu, T., Nishiyama, S., et al. 2009, MNRAS, 399, 1709

Matsunaga, N., Feast, M. W., Kawadu, T., et al. 2013, MNRAS, 429, 385

Matteucci, F., \& François, P. 1989, MNRAS, 239, 885

Merle, T., Thévenin, F., Pichon, B., \& Bigot, L. 2011, MNRAS, 418, 863

Minchev, I., \& Famaey, B. 2010, ApJ, 722, 112

Minchev, I., Famaey, B., Combes, F., et al. 2011, A\&A, 527, A147

Minchev, I., Chiappini, C., \& Martig, M. 2013, A\&A, 558, A9

Mollá, M., \& Díaz, A. I. 2005, MNRAS, 358, 521

Monson, A. J., \& Pierce, M. J. 2011, ApJS, 193, 12

Ngeow, C.-C. 2012, ApJ, 747, 50

Ngeow, C.-C., \& Kanbur, S. M. 2005, MNRAS, 360, 1033
Nordström, B., Mayor, M., Andersen, J., et al. 2004, A\&A, 418, 989

Pancino, E., Carrera, R., Rossetti, E., \& Gallart, C. 2010, A\&A, 511, A56

Pedicelli, S., Bono, G., Lemasle, B., et al. 2009, A\&A, 504, 81

Pedicelli, S., Lemasle, B., Groenewegen, M., et al. 2010, A\&A, 518, A11

Perinotto, M., \& Morbidelli, L. 2006, MNRAS 372, 45

Pilkington, K., Few, C. G., Gibson, B. K., et al. 2012, A\&A, 540, A56

Piovan, L., Chiosi, C., Merlin, E., et al. 2011, unpublished [arXiv: 1107.4567]

Portinari, L., \& Chiosi, C. 2000, A\&A, 355, 929

Pottasch, S. R., \& Bernard-Salas, J. 2006, A\&A, 457, 189

Prantzos, N., \& Boissier, S. 2000, MNRAS, 313, 338

Quillen, A. C., Minchev, I., Bland-Hawthorn, J., \& Haywood, M. 2009, MNRAS, 397, 1599

Quireza, C., Rood, R. T., Bania, T. M., Balser, D. S., \& Maciel, W. J. 2006b, ApJ, 653, 1226

Rahimi, A., Kawata, D., Allende Prieto, C., et al. 2011, MNRAS, 415, 1469

Recio-Blanco, A., Bijaoui, A., \& de Laverny, P. 2006, MNRAS, 370, 141

Reid, M. J., Menten, K. M., Zheng, X. W., Brunthaler, A., \& Xu, Y. 2009, ApJ, 705,1548

Rolleston, W. R. J., Smartt, S. J., Dufton, P. L., \& Ryans, R. S. I. 2000, A\&A, 363,537

Romaniello, M., Primas, F., Mottini, M., et al. 2008, A\&A, 488, 731

Roškar, R., Debattista, V. P., Stinson, G. S., et al. 2008a, ApJ, 675, 65

Roškar, R., Debattista, V. P., Quinn, T. R., Stinson, G. S., \& Wadsley, J. 2008b, ApJ, 684, 79

Rudolph, A. L., Fich, M., Bell, G. R., et al. 2006, ApJS, 162, 346

Ryabchikova, T. A., Piskunov, N. E., Stempels, H. C., Kupka, F., \& Weiss, W. W. 1999, Phys. Scr., T83, 162

Sanders, N., Caldwell, N., \& McDowell, J. 2010, BAAS, 36, 1113

Sasselov, D. D. 1986, PASP, 98, 561

Scarano, S., \& Lépine, J. R. D. 2013, MNRAS, 428, 625

Schönrich, R., \& Binney, J. 2009, MNRAS, 396, 203

Sellwood, J. A., \& Binney, J. J. 2002, MNRAS, 336, 785

Sestito, P., Bragaglia, A., Randich, S., et al. 2006, A\&A, 458, 121

Sestito, P., Randich, S., \& Bragaglia, A. 2007, A\&A, 465, 185

Sestito, P., Bragaglia, A., Randich, S., et al. 2008, A\&A, 488, 943

Shappee, B. J., \& Stanek, K. Z. 2011, ApJ, 733, 124

Simpson, J. P., Colgan, S. W. J., Rubin, R. H., Erickson, E. F., \& Haas, M. R. 1995, ApJ, 444, 721

Smartt, S. J., \& Rolleston, W. R. J. 1997, ApJ, 481, L47

Soszyński, I., Gieren, W., \& Pietrzyński, G. 2005, PASP, 117, 823

Spitoni, E., \& Matteucci, F. 2011, A\&A, 531, 72

Spitzer, L., \& Schwarzschild, M. 1953, ApJ, 118, 106

Stanghellini, L., \& Haywood, M. 2010, ApJ, 714, 1096

Stanghellini, L., Guerrero, M. A., Cunha, K., Manchado, A., \& Villaver, E. 2006, ApJ, 651, 898

Steinmetz, M., Zwitter, T., Siebert, A., et al. 2006, AJ, 132, 1645

Stinson, G. S., Bailin, J., Couchman, H., et al. 2010, MNRAS, 408, 812

Storm, J., Gieren, W., Fouqué, P., et al. 2011a, A\&A, 534, A94

Storm, J., Gieren, W., Fouqué, P., et al. 2011b, A\&A, 534, A95

Takeda, Y., Kang, D.-I., Han, I., Lee, B.-C., \& Kim, K.-M. 2013, MNRAS, 432, 769

Tammann, G. A., Sandage, A., \& Reindl, B. 2003, A\&A, 404, 423

Trippe, S., Gillessen, S., Gerhard, O. E., et al. 2008, A\&A, 492, 419

Twarog, B. A., Ashman, K. M., \& Antony-Twarog, B. J. 1997, AJ, 114, 2556

Usenko, I. A., Kniazev, A. Y., Berdnikov, L. N., \& Kravtsov, V. V. 2011a, Astron. Lett., 37, 499

Usenko, I. A., Berdnikov, L. N., Kravtsov, V. V., et al. 2011b, Astron. Lett., 37, 718

Vilchez, J. M., \& Esteban, C. 1996, MNRAS, 280, 720

Vlajić, M., Bland-Hawthorn, J., \& Freeman, K. C. 2009, ApJ, 697, 361

Vlajić, M., Bland-Hawthorn, J., \& Freeman, K. C. 2011, ApJ, 732, 7

Wang, Y., \& Zhao, G. 2013, ApJ, 769, 4

Wiersma, R. P. C., Schaye, J., \& Theuns, T. 2011, MNRAS, 415, 353

Yanny, B., Rockosi, C., Newberg, H. J., Knapp, G. R., et al. 2009, AJ, 137, 4377

Yong, D., Carney, B. W., \& de Almeida, M. L. T. 2005, AJ, 130, 597

Yong, D., Carney, B. W., de Almeida, M. L. T., \& Pohl, B. L. 2006, AJ, 131, 2256

Yong, D., Carney, B. W., \& Friel, E. D. 2012, AJ, 144, 95 


\section{Appendix A: Line list}

Table A.1. List of the absorption lines used for the determination of the abundances.

\begin{tabular}{|c|c|c|c|}
\hline $\begin{array}{l}\text { Wavelength } \\
\AA\end{array}$ & Element & $\begin{array}{l}\chi_{\mathrm{ex}} \\
\mathrm{eV}\end{array}$ & $\log g f$ \\
\hline 7835.31 & Al I & 4.02 & -0.65 \\
\hline 7836.13 & $\mathrm{Al} \mathrm{I}$ & 4.02 & -0.49 \\
\hline 4486.91 & Ce II & 0.29 & -0.26 \\
\hline 4554.04 & Ba II & 0.00 & 0.17 \\
\hline 4562.37 & Ce II & 0.48 & 0.23 \\
\hline 4578.56 & $\mathrm{Ca} \mathrm{I}$ & 2.52 & -0.70 \\
\hline 4685.27 & Ca I & 2.93 & -0.88 \\
\hline 4721.02 & Ca II & 7.05 & -0.78 \\
\hline 4959.12 & Nd II & 0.06 & -0.80 \\
\hline 5044.02 & Ce I & 1.21 & -0.07 \\
\hline 5092.79 & Nd II & 0.38 & -0.61 \\
\hline 5112.28 & $\mathrm{Zr}$ II & 1.66 & -0.85 \\
\hline 5114.56 & La II & 0.23 & -1.03 \\
\hline 5119.12 & Y II & 0.99 & -1.36 \\
\hline 5130.59 & Nd II & 1.30 & 0.45 \\
\hline 5181.17 & Nd II & 0.86 & -0.74 \\
\hline 5289.81 & Y II & 1.03 & -1.85 \\
\hline 5290.82 & La II & 0.00 & -1.65 \\
\hline 5349.47 & $\mathrm{Ca} \mathrm{I}$ & 2.71 & -0.31 \\
\hline 5402.77 & Y II & 1.84 & -0.63 \\
\hline 5431.52 & $\mathrm{Nd}$ II & 1.12 & -0.47 \\
\hline 5509.91 & Y II & 0.99 & -0.95 \\
\hline 5518.49 & $\mathrm{Ce}$ II & 1.15 & -0.67 \\
\hline 5581.97 & Ca I & 2.52 & -0.55 \\
\hline 5590.11 & $\mathrm{Ca} \mathrm{I}$ & 2.52 & -0.57 \\
\hline 5601.28 & $\mathrm{Ca} \mathrm{I}$ & 2.53 & -0.52 \\
\hline 5665.56 & Si I & 4.92 & -1.94 \\
\hline 5688.21 & $\mathrm{Na} \mathrm{I}$ & 2.10 & -0.40 \\
\hline 5690.43 & Si I & 4.93 & -1.77 \\
\hline 5711.09 & Mg I & 4.35 & -1.83 \\
\hline 5728.89 & Y II & 1.84 & -1.12 \\
\hline 5805.77 & La II & 0.13 & -1.56 \\
\hline 5853.69 & Ba II & 0.60 & -0.91 \\
\hline 5867.56 & Ca I & 2.93 & -1.57 \\
\hline 5948.54 & Si I & 5.08 & -1.13 \\
\hline 6043.39 & Ce II & 1.21 & -0.48 \\
\hline 6125.03 & Si I & 5.61 & -1.46 \\
\hline 6141.71 & Ba II & 0.70 & -0.03 \\
\hline 6154.23 & $\mathrm{Na} \mathrm{I}$ & 2.10 & -1.55 \\
\hline 6155.14 & Si I & 5.62 & -0.75 \\
\hline 6160.75 & $\mathrm{Na} \mathrm{I}$ & 2.10 & -1.25 \\
\hline 6166.44 & Ca I & 2.52 & -1.14 \\
\hline 6244.48 & Si I & 5.62 & -1.09 \\
\hline 6262.29 & La II & 0.40 & -1.22 \\
\hline 6300.30 & O I & 0.00 & -9.71 \\
\hline 6363.77 & O I & 0.02 & -10.2 \\
\hline 6390.48 & La II & 0.32 & -1.41 \\
\hline 6414.98 & Si I & 5.87 & -1.03 \\
\hline 6437.64 & Eu II & 1.32 & -0.32 \\
\hline 6471.66 & $\mathrm{Ca} \mathrm{I}$ & 2.53 & -0.69 \\
\hline 6496.89 & Ba II & 0.60 & -0.41 \\
\hline 6538.60 & S I & 8.05 & -0.93 \\
\hline 6645.13 & Eu II & 1.38 & 0.12 \\
\hline 6696.02 & Al I & 3.14 & -1.57 \\
\hline 6698.67 & Al I & 3.14 & -1.87 \\
\hline 6740.08 & Nd II & 0.06 & -1.53 \\
\hline 6741.63 & Si I & 5.98 & -1.65 \\
\hline 6748.84 & S I & 7.87 & -0.60 \\
\hline
\end{tabular}

Notes. The list is ordered by wavelength and also displays the excitation potential $\left(\chi_{\mathrm{ex}}\right)$ and the logarithm of the weighted oscillator strength $(\log g f)$ of the transition.
Table A.1. continued.

\begin{tabular}{lccr}
\hline \hline $\begin{array}{l}\text { Wavelength } \\
\AA\end{array}$ & Element & $\begin{array}{c}\chi_{\mathrm{ex}} \\
\mathrm{eV}\end{array}$ & $\log g f$ \\
\hline 6757.17 & S I & 7.87 & -0.31 \\
6774.27 & La II & 0.13 & -1.71 \\
6795.41 & Y II & 1.74 & -1.03 \\
7034.90 & Si I & 5.87 & -0.88 \\
7375.25 & Si I & 6.19 & -1.05 \\
7423.50 & Si I & 5.62 & -0.18 \\
7680.27 & Si I & 5.86 & -0.69 \\
7849.97 & Si I & 6.19 & -0.72 \\
7881.88 & Y II & 1.84 & -0.57 \\
7932.35 & Si I & 5.96 & -0.47 \\
7944.00 & Si I & 5.98 & -0.31 \\
8712.69 & Mg I & 5.93 & -1.37 \\
8736.02 & Mg I & 5.95 & -0.36 \\
8772.87 & Al I & 4.02 & -0.17 \\
8773.90 & Al I & 4.02 & -0.02 \\
9237.54 & S I & 6.53 & 0.04 \\
9276.97 & Zr I & 0.69 & -1.00 \\
\hline
\end{tabular}


B. Lemasle et al.: Galactic abundance gradients from Cepheids

Appendix B: Individual abundance results

Table B.1. Light and $\alpha$ element abundance measurements for the Cepheids in our sample.

\begin{tabular}{|c|c|c|c|c|c|c|c|}
\hline Star & $\begin{array}{l}\text { Na } \\
\text { dex }\end{array}$ & $\begin{array}{l}\mathrm{Mg} \\
\mathrm{dex} \\
\end{array}$ & $\begin{array}{r}\text { Al } \\
\text { dex }\end{array}$ & $\begin{array}{c}\mathrm{Si} \\
\mathrm{dex} \\
\end{array}$ & $\begin{array}{c}\mathrm{S} \\
\mathrm{dex} \\
\end{array}$ & $\begin{array}{c}\mathrm{Ca} \\
\mathrm{dex} \\
\end{array}$ & $\begin{array}{c}\mathrm{Fe} \\
\mathrm{dex} \\
\end{array}$ \\
\hline AAGem & & $-0.26 \pm 0.00(1)$ & & $-0.24 \pm 0.10(11)$ & $-0.24 \pm 0.00(1)$ & $-0.37 \pm 0.14(2)$ & $-0.35 \pm 0.08 \quad(75)$ \\
\hline ADGem & $-0.05 \pm 0.00$ & $-0.05 \pm 0.05$ & $-0.08 \pm 0.09(4)$ & $-0.11 \pm 0.15(14)$ & $-0.19 \pm 0.00(1)$ & $-0.11 \pm 0.14(6)$ & $-0.19 \pm 0.13$ \\
\hline ADPup & $0.04 \pm 0.13(2)$ & $0.02 \pm 0.12(2)$ & $-0.09 \pm 0.18(6)$ & $-0.10 \pm 0.09(13)$ & $0.05 \pm 0.00$ & $-0.02 \pm 0.09$ & $-0.20 \pm 0.12(114)$ \\
\hline AHVel & $0.53 \pm 0.05(x)$ & $0.14 \pm 0.10(x)$ & $0.16 \pm 0.12(x)$ & $0.12 \pm 0.15(\mathrm{x})$ & $0.24 \pm 0.09(\mathrm{x})$ & $0.04 \pm 0.12(\mathrm{x})$ & $-0.04 \pm 0.06 \quad(\mathrm{x})$ \\
\hline AOAur & $-0.10 \pm 0.00$ & $-0.08 \pm 0.08$ & $-0.11 \pm 0.11$ & $-0.15 \pm 0.16(11)$ & $-0.22 \pm 0.00$ & $-0.18 \pm 0.08$ & $-0.41 \pm 0.11(106)$ \\
\hline AOCMa & $.25 \pm 0.00(1)$ & $0.27 \pm 0.00$ & $0.35 \pm 0.00(1)$ & $0.24 \pm 0.15$ & $0.44 \pm 0.11$ & $-0.01 \pm 0.26(5)$ & $-0.04 \pm 0.13$ \\
\hline APPup & $0.06 \pm 0.01(x)$ & $-0.05 \pm 0.05(\mathrm{x})$ & $-0.11 \pm 0.11(\mathrm{x})$ & $-0.09 \pm 0.12 \quad(\mathrm{x})$ & $0.23 \pm 0.15(x)$ & $-0.14 \pm 0.12(\mathrm{x})$ & $-0.15 \pm 0.08 \quad(x)$ \\
\hline AQCar & & $0.38 \pm 0.00$ & $0.28 \pm 0.10$ & $0.18 \pm 0.19$ & & $0.36 \pm 0.11$ & $-0.30 \pm 0.10(106)$ \\
\hline AQPup & $0.12 \pm 0.00(1)$ & & $-0.02 \pm 0.18$ & $-0.01 \pm 0.10(11)$ & $-0.06 \pm 0.28$ (2) & $-0.13 \pm 0.14$ & $-0.26 \pm 0.03$ \\
\hline ATPup & $0.29 \pm 0.10(2)$ & $0.19 \pm 0.13(2)$ & $-0.20 \pm 0.03$ & $-0.04 \pm 0.18(11)$ & & $-0.15 \pm 0.17$ & $-0.22 \pm 0.10$ \\
\hline & $0.09 \pm 0.00(1)$ & $0.73 \pm 0.00(1)$ & $.59 \pm 0.00(1)$ & $0.41 \pm 0.12$ & $0.75 \pm 0.20(2)$ & $0.43 \pm 0.08$ & $0.26 \pm 0.12 \quad(x)$ \\
\hline AVTau & $0.17 \pm 0.00$ & $0.12 \pm 0.09$ & $0.09 \pm 0.23(3)$ & $0.04 \pm 0.18$ & $0.05 \pm 0.00$ & $-0.03 \pm 0.09$ & $-0.17 \pm 0.11$ \\
\hline AXAur & $0.17 \pm 0.00$ & $-0.01 \pm 0.16(2)$ & $-0.09 \pm 0.08$ (2) & $-0.02 \pm 0.16(13)$ & $-0.08 \pm 0.00$ & $-0.04 \pm 0.16(6)$ & $-0.22 \pm 0.10(109)$ \\
\hline AXVel & & $0.13 \pm 0.00$ & $12 \pm 0.06(2)$ & $0.20 \pm 0.21$ & $0.38 \pm 0.16$ & $0.03 \pm 0.08$ & $-0.15 \pm 0.07$ \\
\hline BEMon & $0.05 \pm 0.00(1)$ & $-0.09 \pm 0.05(2)$ & $0.05 \pm 0.12(4)$ & $-0.05 \pm 0.14(12)$ & $-0.03 \pm 0.00(1)$ & $-0.12 \pm 0.12(5)$ & $-0.07 \pm 0.09$ (106) \\
\hline BGVel & & & $-0.11 \pm 0$. & $0.09 \pm 0.07(12)$ & $0.25 \pm 0.00$ & $0.08 \pm 0$ & $-0.10 \pm 0.08(130)$ \\
\hline BKAur & & $0.32 \pm 0.00(\mathrm{x})$ & -0.0 & $0.16 \pm 0.15 \quad(x)$ & $0.41 \pm 0.09(\mathrm{x})$ & $0.05 \pm 0.14(\mathrm{x})$ & $-0.07 \pm 0.11 \quad(\mathrm{x})$ \\
\hline & $0.32 \pm 0.08(2)$ & $0.16 \pm 0.16(2)$ & & $0.12 \pm 0.18$ & $0.21 \pm 0.00(1)$ & & $-0.03 \pm 0.10(128)$ \\
\hline BVMon & & $0.14 \pm 0$ & $2 \pm 0$ & $0.01 \pm 0.2$ & & $-0.03 \pm 0$ & $-0.10 \pm 0.10$ \\
\hline CSOri & $-0.03 \pm 0.00$ & $-0.18 \pm 0.04$ & $9 \pm 0.00$ & $-0.20 \pm 0.22$ & $-0.29 \pm 0.00(1)$ & $-0.20 \pm 0.09$ & $-0.19 \pm 0.13$ \\
\hline & $19 \pm 0$ & $0.18 \pm 0$ & +0 & $0.07 \pm 0.17$ & $0.05 \pm 0$ & $-0.12 \pm 0$ & $-0.10 \pm 0.13(111)$ \\
\hline & 0.2 & $.21 \pm$ & & (11) & $0.36 \pm$ & $0.26 \pm$ & $-0.01 \pm 0.07(122)$ \\
\hline EKMon & & $0.05 \pm 0.18(2)$ & $2 \pm 0$ & $-0.06 \pm 0.13(11)$ & $-0.15 \pm 0.00$ & $-0.04 \pm 0.0$ & $-0.05 \pm 0.11$ \\
\hline EZVel & $0.10 \pm 0.00(\mathrm{x})$ & $0.32 \pm 0.00(\mathrm{x})$ & & $0.02 \pm 0.15(\mathrm{x})$ & & & $-0.01 \pm 0.11 \quad(\mathrm{x})$ \\
\hline HWPup & & $-0.02 \pm c$ & & $-0.38 \pm 0.10$ & -0.0 & $-0.23 \pm 0$ & $-0.28 \pm 0.18$ \\
\hline lCar & & & $0.11 \pm 0$ & $0.20 \pm 0.11(\mathrm{x})$ & & $-0.00 \pm 0.00(\mathrm{x})$ & $0.10 \pm 0.20 \quad(\mathrm{x})$ \\
\hline & $0.13 \pm 0.01(2)$ & $-0.18 \pm 0.01(2)$ & & $-0.06 \pm 0.08(15)$ & $-0.07 \pm 0.11(2)$ & & $-0.25 \pm 0.08$ \\
\hline & 0 & $0.10 \pm$ & 0. & $0.12 \pm 0.10$ & $0.29 \pm c$ & $6 \pm 0$ & $-0.14 \pm 0.13(105)$ \\
\hline & & $0.21 \pm 0$ & & $8(10)$ & $0.28 \pm 0$ & & $0.07 \pm 0.09$ \\
\hline & 0 & $0.07 \pm 0.00(\mathrm{x})$ & $12+8$ & $0.07 \pm 0.11 \quad(\mathrm{x})$ & $0.58 \pm 0$ & $0.02 \pm$ & $-0.16 \pm 0.09 \quad(\mathrm{x})$ \\
\hline RYVel & $0.44 \pm 0.0$ & & $0.40 \pm 0.08$ & $0.18 \pm 0.12$ & $0.48 \pm 0$ & $0.13 \pm 0$ & $-0.05 \pm 0.08$ \\
\hline & $06 \pm 0$ & $0.12 \pm 0.00(1)$ & & & $0.16 \pm 0$ & & $-0.20 \pm 0.07$ \\
\hline RZGem & & & $0.04 \pm 0.18$ & $-0.14 \pm 0.20(11)$ & $020=0$ & $-0.22 \pm 0$. & $-0.44 \pm 0.07$ \\
\hline RZVel & & $0.43 \pm 0.00(1)$ & & $0.28 \pm 0.12$ & & $-0.23 \pm 0.25(2)$ & $0.05 \pm 0.10$ \\
\hline & & $0.08 \pm$ & & $8 \pm 0.18$ & $0.17 \pm 0.00(1)$ & & $-0.14 \pm 0.10$ \\
\hline & & $0.26 \pm 0.00(1)$ & 4) & $0.12 \pm 0.11(12)$ & & $0.09 \pm 0.10$ & $-0.14 \pm 0.10$ \\
\hline SVMon & & $-0.08 \pm 0.00$ & $2 \pm 0.35(2)$ & $-0.02 \pm 0.10$ & & $-0.27 \pm 0.00$ & $-0.10 \pm 0.14$ \\
\hline & & $0.28 \pm 0$. & & $-0.03 \pm 0.10$ & & & $5 \pm 0.08 \quad(66)$ \\
\hline & & & 3) & $4(13)$ & $0.39 \pm 0.00(1)$ & $-0.01 \pm 0$. & $-0.18 \pm 0.07$ (114) \\
\hline & & & & & & & $-0.13 \pm 0.11$ \\
\hline & & 0 & $9 \pm 0$ & $0.28 \pm 0.18$ & $0.25 \pm 0.00(1)$ & (6) & $-0.02 \pm 0.07(144)$ \\
\hline TWCMa & & $-0.23 \pm 0.00$ & $0.07 \pm 0.18$ & $-0.31 \pm 0.09$ & & $-0.40 \pm 0$ & $-0.51 \pm 0.09(112)$ \\
\hline & & & & $0.14 \pm 0.19$ & $0.05 \pm 0.00$ & & $-0.15 \pm 0.08$ \\
\hline & $0.07 \pm 0.00$ & $-0.12 \pm 0$ & ) & $6 \pm 0.10(14)$ & $-0.23 \pm c$ & (8) & $-0.12 \pm 0.10$ \\
\hline & $24+00081$ & $-0.09 \pm 0.14$ & $-0.05 \pm 0.14$ & $-0.21 \pm 0.04(14)$ & $-0.21 \pm 0$ & $-0.11 \pm 0.1$ & $-0.15 \pm 0.11(104)$ \\
\hline & $0.10 \pm 0.00(1)$ & & $-0.06 \pm 0$. & $-0.01 \pm 0.11(13)$ & $0.09 \pm 0$ & & $-0.04 \pm 0.08$ \\
\hline & & $-0.06 \pm 0$ & ) & $5 \pm 0.06$ & $0.41 \pm 0$ & $0.14 \pm 0$ & $-0.10 \pm 0.07$ (111) \\
\hline UYMon & $0.08 \pm 0.05(2)$ & $-0.23 \pm 0.06(2)$ & $-0.25 \pm 0.18$ & $-0.22 \pm 0.15(15)$ & $-0.15 \pm 0.11(2)$ & $-0.28 \pm 0.15$ & $-0.33 \pm 0.08$ \\
\hline & & $0.40 \pm 0.00$ & & $0.47 \pm 0.12$ & & $0.23 \pm 0.08$ & $0.35 \pm 0.17 \quad(\mathrm{x})$ \\
\hline & & & $0.47 \pm 0.00(1)$ & $0.44 \pm 0.05$ & $0.80 \pm 0.00$ & & $0.38 \pm 0.17 \quad(\mathrm{x})$ \\
\hline & & & $0.21 \pm 0.09(2)$ & $0.20 \pm 0.16$ & & & $-0.08 \pm 0.09(113)$ \\
\hline & & $0.23 \pm 0.10(2)$ & & $1 \pm 0.17$ & $-0.04 \pm$ & & $-0.17 \pm 0.15$ \\
\hline & & & & $-0.01 \pm 0.17(11)$ & $-0.10 \pm 0.00$ & $-0.09 \pm 0.18$ & $-0.25 \pm 0.07$ \\
\hline & & & & 13 (7) & & & $-0.12 \pm 0.13$ \\
\hline VCar & & & & $2(12)$ & $0.26 \pm 0$ & $.13(3)$ & $-0.06 \pm 0.07$ (114) \\
\hline VVel & $-0.01 \pm 0.00(1)$ & $0.05 \pm 0.04$ & $-0.06 \pm 0.24$ & $-0.12 \pm 0.10(10)$ & $-0.19 \pm 0.00(1)$ & $-0.11 \pm 0.10$ & $-0.30 \pm 0.06(113)$ \\
\hline & & $-0.11 \pm 0$. & $-0.16 \pm 0.14(6)$ & $-0.12 \pm 0.17(11)$ & & $-0.10 \pm 0.12(5)$ & $-0.15 \pm 0.12(137)$ \\
\hline & $9+0$ & & & $0.49 \pm 0.18$ & $0.26 \pm 0.00(1)$ & $0.16 \pm 0.10$ & $-0.06 \pm 0.06$ \\
\hline VYSgr & $6 \pm 0.13(2)$ & $0.47 \pm 0.00$ & $0.20 \pm 0.12(2)$ & $0.37 \pm 0.05$ & & $0.22 \pm 0.10(5)$ & $0.38 \pm 0.17 \quad(x)$ \\
\hline & & & & $-0.21 \pm 0.12$ & & $-0.35 \pm 0.14(5)$ & $-0.37 \pm 0.07(115)$ \\
\hline WWMon & $0.13 \pm 0.00(1)$ & $-0.09 \pm 0.24$ & & $-0.02 \pm 0.14$ & $08(2)$ & $-0.26 \pm 0.11$ & $-0.32 \pm 0.13$ \\
\hline & $0.22 \pm 0.02(2)$ & & $-0.02 \pm 0$ & $-0.12 \pm 0.14(10)$ & $-0.13 \pm 0.28(2)$ & $-0.04 \pm 0.08(5)$ & $-0.15 \pm 0.09(110)$ \\
\hline & & & & $-0.12 \pm 0.13(12)$ & $-0.19 \pm 0.22$ & & $-0.18 \pm 0.11$ \\
\hline YAur & $0.27 \pm 0.00(1)$ & $-0.01 \pm 0.21(2)$ & $-0.11 \pm 0.20(5)$ & $-0.01 \pm 0.20(13)$ & $0.22 \pm 0.02$ & $-0.17 \pm 0.10(6)$ & $-0.26 \pm 0.12$ \\
\hline YZAur & $0.22 \pm 0.00(1)$ & $-0.20 \pm 0.00$ & & $-0.13 \pm 0.12(11)$ & $-0.23 \pm 0.00$ & $-0.14 \pm 0.10$ & $-0.33 \pm 0.12$ \\
\hline
\end{tabular}

Notes. The abundances are tabulated together with the rms uncertainties and with the number of lines used. (x) indicates that the final abundance is the mean value of the abundances derived from several spectra. 
Table B.2. Same as Table B.1, but for heavy elements.

\begin{tabular}{|c|c|c|c|c|c|c|}
\hline Star & $\begin{array}{c}\mathrm{Y} \\
\operatorname{dex}\end{array}$ & $\begin{array}{c}\mathrm{Zr} \\
\mathrm{dex}\end{array}$ & $\begin{array}{l}\text { La } \\
\text { dex }\end{array}$ & $\begin{array}{c}\mathrm{Ce} \\
\mathrm{dex}\end{array}$ & $\begin{array}{l}\mathrm{Nd} \\
\mathrm{dex}\end{array}$ & $\begin{array}{l}\mathrm{Eu} \\
\mathrm{dex}\end{array}$ \\
\hline AAGem & $-0.33 \pm 0.10(5)$ & $-0.15 \pm 0.00(1)$ & $-0.11 \pm 0.05$ (5) & $-0.08 \pm 0.08(2)$ & $-0.06 \pm 0.14(3)$ & $-0.09 \pm 0.06(2)$ \\
\hline ADGem & $-0.17 \pm 0.06(6)$ & $-0.02 \pm 0.00$ & $0.06 \pm 0.05(6)$ & $0.26 \pm 0.06$ & $0.10 \pm 0.07$ & $0.05 \pm 0.04$ \\
\hline ADPup & $0.23 \pm 0.21(5)$ & $0.16 \pm 0.00$ & $0.42 \pm 0.26$ & $0.31 \pm 0.13(2)$ & $0.50 \pm 0.19(6)$ & $0.17 \pm 0.13(2)$ \\
\hline AHVel & $0.11 \pm 0.09(\mathrm{x})$ & $0.22 \pm 0.00(\mathrm{x})$ & $0.26 \pm 0.15(\mathrm{x})$ & $0.33 \pm 0.07(\mathrm{x})$ & $0.30 \pm 0.16(\mathrm{x})$ & $0.30 \pm 0.00(\mathrm{x})$ \\
\hline AOAur & $-0.40 \pm 0.11(5)$ & $-0.35 \pm 0.00(1)$ & $-0.21 \pm 0.17(6)$ & $-0.17 \pm 0.23(2)$ & $-0.22 \pm 0.23(6)$ & $-0.15 \pm 0.00$ \\
\hline AOCMa & $0.03 \pm 0.04$ & & $0.29 \pm 0.13$ & & & \\
\hline APPup & $0.11 \pm 0.09(\mathrm{x})$ & $0.19 \pm 0.00(\mathrm{x})$ & $0.30 \pm 0.12(\mathrm{x})$ & $0.35 \pm 0.05(\mathrm{x})$ & $0.33 \pm 0.14(\mathrm{x})$ & $0.32 \pm 0.09(\mathrm{x})$ \\
\hline AQCar & $-0.02 \pm 0.15(6)$ & & $0.02 \pm 0.21(4)$ & $0.02 \pm 0.00(1)$ & $0.25 \pm 0.16(2)$ & \\
\hline AQPup & $0.03 \pm 0.05$ & & $0.08 \pm 0.20$ & $0.09 \pm 0.04$ & $0.02 \pm 0.20(3)$ & $0.03 \pm 0.18$ \\
\hline ATPup & $-0.19 \pm 0.10(5)$ & $-0.12 \pm 0.00$ & $-0.11 \pm 0.18$ & $0.06 \pm 0.01(2)$ & $0.06 \pm 0.18$ & $-0.21 \pm 0.00$ \\
\hline AVSgr & $0.10 \pm 0.02$ & & $0.05 \pm 0.06$ & $0.12 \pm 0.04(2)$ & & $0.57 \pm 0.16$ \\
\hline AVTau & $-0.08 \pm 0.09$ (4) & $-0.13 \pm 0.00$ & $0.08 \pm 0.08$ & & $0.28 \pm 0.00$ & $0.16 \pm 0.40(2)$ \\
\hline AXAur & $-0.35 \pm 0.14(6)$ & $-0.22 \pm 0.00(1)$ & $-0.22 \pm 0.10(6)$ & $-0.08 \pm 0.05$ & $-0.10 \pm 0.10$ & $-0.31 \pm 0.06(2)$ \\
\hline AXVel & $0.02 \pm 0.07$ & $0.03 \pm 0.00$ & $0.28 \pm 0.15$ & $0.44 \pm 0.16$ & $0.16 \pm 0.01$ & $0.14 \pm 0.00$ \\
\hline BEMon & $0.06 \pm 0.12$ & $-0.04 \pm 0.00(1)$ & $0.11 \pm 0.08$ & $0.11 \pm 0.15$ & $0.05 \pm 0.08$ & $0.03 \pm 0.12(2)$ \\
\hline BGVel & $0.08 \pm 0.13(6)$ & $0.17 \pm 0.00$ & $0.24 \pm 0.13$ & $0.35 \pm 0.17$ & $0.27 \pm 0.18$ & $0.21 \pm 0.00$ \\
\hline BKAur & $0.08 \pm 0.15(\mathrm{x})$ & $0.14 \pm 0.00(\mathrm{x})$ & $0.26 \pm 0.08(\mathrm{x})$ & $0.31 \pm 0.07(\mathrm{x})$ & $0.28 \pm 0.11(\mathrm{x})$ & $0.27 \pm 0.02(x)$ \\
\hline BNPup & $0.26 \pm 0.16(5)$ & & $0.39 \pm 0.16$ & $0.43 \pm 0.04$ & $0.52 \pm 0.18$ & $0.28 \pm 0.05(2)$ \\
\hline BVMon & $-0.12 \pm 0.24(2)$ & & $0.24 \pm 0.13(2)$ & & & $0.27 \pm 0.00(1)$ \\
\hline CSOri & $-0.20 \pm 0.14$ & $0.15 \pm 0.00$ & & $0.14 \pm 0.00(1)$ & $0.14 \pm 0.28(2)$ & \\
\hline CVMon & $-0.09 \pm 0.07(7)$ & $0.04 \pm 0.00(1)$ & $0.06 \pm 0.03$ & $0.08 \pm 0.14$ & $0.07 \pm 0.12$ & $0.03 \pm 0.04$ \\
\hline DRVel & $0.13 \pm 0.10$ & $0.40 \pm 0.00$ & $0.14 \pm 0.23$ & $0.29 \pm 0.28(2)$ & $0.17 \pm 0.33$ & $-0.04 \pm 0.00$ \\
\hline EKMon & $-0.19 \pm 0.11$ & $0.12 \pm 0.00$ & $-0.05 \pm 0.08$ & $0.18 \pm 0.16(3)$ & $0.16 \pm 0.06$ & $0.01 \pm 0.00$ \\
\hline EZVel & $0.20 \pm 0.21(\mathrm{x})$ & & $0.51 \pm 0.15(\mathrm{x})$ & $0.47 \pm 0.09(\mathrm{x})$ & $0.12 \pm 0.22(\mathrm{x})$ & $0.61 \pm 0.28(\mathrm{x})$ \\
\hline HWPup & $-0.42 \pm 0.18$ & $-0.36 \pm 0.00(1)$ & $-0.21 \pm 0.17(5)$ & $-0.04 \pm 0.00(1)$ & $-0.20 \pm 0.11$ & $-0.13 \pm 0.13(2)$ \\
\hline 1Car & $0.29 \pm 0.07(\mathrm{x})$ & & $0.29 \pm 0.19(\mathrm{x})$ & $0.42 \pm 0.13(\mathrm{x})$ & $0.47 \pm 0.16(\mathrm{x})$ & $0.61 \pm 0.00(\mathrm{x})$ \\
\hline MYPup & $0.01 \pm 0.10(6)$ & $0.07 \pm 0.00$ & $0.17 \pm 0.13(6)$ & $0.25 \pm 0.15$ & $0.16 \pm 0.19$ & $0.16 \pm 0.08(2)$ \\
\hline RSOri & $0.13 \pm 0.16(5)$ & $0.23 \pm 0.00$ & $0.30 \pm 0.14$ & $0.39 \pm 0.00$ & $0.36 \pm 0.23$ & $0.31 \pm 0.06(2)$ \\
\hline RSPup & $0.30 \pm 0.20$ & & $0.24 \pm 0.16$ & $0.19 \pm 0.09(2)$ & $0.39 \pm 0.32$ & $0.37 \pm 0.16(2)$ \\
\hline RYCMa & $-0.08 \pm 0.12(\mathrm{x})$ & $0.14 \pm 0.00(\mathrm{x})$ & $0.13 \pm 0.16(x)$ & $0.20 \pm 0.07(\mathrm{x})$ & $0.19 \pm 0.19(\mathrm{x})$ & $0.28 \pm 0.00(\mathrm{x})$ \\
\hline RYVel & $0.19 \pm 0.22$ & & $0.07 \pm 0.37$ & $0.15 \pm 0.28$ & $0.17 \pm 0.25$ & $0.50 \pm 0.20(2)$ \\
\hline RZCMa & $-0.16 \pm 0.16(6)$ & $-0.12 \pm 0.00(1)$ & $-0.07 \pm 0.16$ & $0.09 \pm 0.04$ & $0.14 \pm 0.09$ & $0.09 \pm 0.00$ \\
\hline RZGem & $-0.41 \pm 0.19(6)$ & $-0.36 \pm 0.00$ & $-0.27 \pm 0.06(5)$ & $-0.02 \pm 0.15$ & $-0.19 \pm 0.08$ & $-0.27 \pm 0.04$ \\
\hline RZVel & $0.34 \pm 0.19$ & & $0.27 \pm 0.24$ & $0.25 \pm 0.12(2)$ & $0.54 \pm 0.00$ & $0.37 \pm 0.00$ \\
\hline STTau & $-0.01 \pm 0.08$ & $0.12 \pm 0.00$ & $0.09 \pm 0.10(5)$ & $0.37 \pm 0.11$ & $0.19 \pm 0.16(3)$ & $0.12 \pm 0.06(2)$ \\
\hline STVel & $-0.04 \pm 0.08(7)$ & & $0.12 \pm 0.12$ & $0.17 \pm 0.00(1)$ & $0.16 \pm 0.19$ & $0.20 \pm 0.00(1)$ \\
\hline SVMon & $0.03 \pm 0.15$ & $0.36 \pm 0.00$ & $0.22 \pm 0.02$ & $0.12 \pm 0.16(2)$ & $0.20 \pm 0.16(2)$ & $0.18 \pm 0.00(1)$ \\
\hline SWVel & $-0.03 \pm 0.00(2)$ & & $0.10 \pm 0.18$ & $0.04 \pm 0.00$ & $-0.02 \pm 0.00(1)$ & $0.08 \pm 0.00(1)$ \\
\hline SXVel & $0.01 \pm 0.15(6)$ & $0.17 \pm 0.00$ & $0.15 \pm 0.09$ & $0.19 \pm 0.02(2)$ & $0.05 \pm 0.11$ & $0.19 \pm 0.00(1)$ \\
\hline SYAur & $-0.07 \pm 0.17(6)$ & & $0.04 \pm 0.13$ & $0.22 \pm 0.05(2)$ & $0.17 \pm 0.11$ & $0.14 \pm 0.10(2)$ \\
\hline TVel & $0.30 \pm 0.05$ & $0.42 \pm 0.00$ & $0.49 \pm 0.12$ & $0.63 \pm 0.20$ & $0.39 \pm 0.16$ & $0.45 \pm 0.00(1)$ \\
\hline TWCMa & $-0.32 \pm 0.07(6)$ & & $-0.03 \pm 0.18$ & & $-0.10 \pm 0.07$ & $0.05 \pm 0.00$ \\
\hline TWMon & $0.21 \pm 0.07$ & & $0.20 \pm 0.07$ & & & \\
\hline TXMon & $-0.12 \pm 0.09(6)$ & $-0.02 \pm 0.00(1)$ & $0.09 \pm 0.11$ & $0.04 \pm 0.09$ & $0.04 \pm 0.07$ & $-0.02 \pm 0.01(2)$ \\
\hline TYMon & $-0.11 \pm 0.13(6)$ & $-0.05 \pm 0.00(1)$ & $-0.05 \pm 0.17(5)$ & $0.02 \pm 0.00(1)$ & $0.07 \pm 0.12(5)$ & $0.09 \pm 0.01(2)$ \\
\hline TZMon & $-0.05 \pm 0.17(6)$ & $0.01 \pm 0.00$ & $0.01 \pm 0.08(6)$ & $0.16 \pm 0.19$ & $0.24 \pm 0.25$ & $0.06 \pm 0.16(2)$ \\
\hline UXCar & $-0.01 \pm 0.10(5)$ & $0.21 \pm 0.00$ & $0.11 \pm 0.04$ & $0.25 \pm 0.09$ & $0.18 \pm 0.21$ & $0.14 \pm 0.00$ \\
\hline UYMon & $-0.35 \pm 0.11(6)$ & $-0.15 \pm 0.00$ & $-0.12 \pm 0.05(6)$ & $-0.04 \pm 0.06(3)$ & $0.01 \pm 0.16(5)$ & $-0.11 \pm 0.06(2)$ \\
\hline UZSct & $0.37 \pm 0.25$ & & $0.48 \pm 0.10$ & $0.57 \pm 0.16(2)$ & & $0.66 \pm 0.00$ \\
\hline V340Ara & & & $0.22 \pm 0.12(2)$ & & & $0.32 \pm 0.00(1)$ \\
\hline V397Car & $-0.04 \pm 0.06$ & & $0.06 \pm 0.13$ & $0.06 \pm 0.00(1)$ & $0.02 \pm 0.11$ & $0.12 \pm 0.00(1)$ \\
\hline V495Mon & & & & & $0.36 \pm 0.00(1)$ & $0.18 \pm 0.00(1)$ \\
\hline V508Mon & $-0.14 \pm 0.13(6)$ & $-0.12 \pm 0.00$ & $0.05 \pm 0.02$ & $0.13 \pm 0.13$ & $0.02 \pm 0.12$ & $0.15 \pm 0.00$ \\
\hline V510Mon & $-0.17 \pm 0.23(3)$ & & & $0.33 \pm 0.12(2)$ & & $0.02 \pm 0.04(2)$ \\
\hline VCar & $0.07 \pm 0.12$ & $0.29 \pm 0.00$ & $0.26 \pm 0.17(5)$ & $0.15 \pm 0.25(2)$ & $0.16 \pm 0.27(5)$ & $0.38 \pm 0.00$ \\
\hline VVel & $-0.13 \pm 0.13(7)$ & $-0.02 \pm 0.00(1)$ & $0.07 \pm 0.14(5)$ & $0.24 \pm 0.13$ & $0.19 \pm 0.09$ & $-0.04 \pm 0.00(1)$ \\
\hline VXPup & $0.04 \pm 0.12$ & $0.02 \pm 0.00$ & $0.32 \pm 0.09(6)$ & $0.35 \pm 0.01$ & $0.26 \pm 0.19$ & $0.23 \pm 0.13$ \\
\hline VYCar & $0.20 \pm 0.09$ & $0.34 \pm 0.00$ & $0.32 \pm 0.12(5)$ & $0.39 \pm 0.01(2)$ & $0.38 \pm 0.23(2)$ & $0.39 \pm 0.00(1)$ \\
\hline VYSgr & $0.23 \pm 0.15$ & & $0.42 \pm 0.29$ & $0.31 \pm 0.23(2)$ & $0.13 \pm 0.00$ & $0.50 \pm 0.07(2)$ \\
\hline VZPup & $-0.08 \pm 0.18$ & $0.10 \pm 0.00$ & $0.07 \pm 0.12$ & $0.14 \pm 0.25$ (2) & $-0.09 \pm 0.12(4)$ & $-0.04 \pm 0.05(2)$ \\
\hline WWMon & $-0.26 \pm 0.17$ & $0.02 \pm 0.00$ & $-0.23 \pm 0.06(2)$ & $-0.21 \pm 0.00$ & $-0.14 \pm 0.22(2)$ & $-0.02 \pm 0.00(1)$ \\
\hline WXPup & $-0.01 \pm 0.10$ & $0.21 \pm 0.00$ & $0.13 \pm 0.25$ & $0.18 \pm 0.01$ & $0.24 \pm 0.21$ & $-0.05 \pm 0.04$ \\
\hline XXMon & $-0.23 \pm 0.18(5)$ & $-0.10 \pm 0.00$ & $0.05 \pm 0.13$ & & $0.03 \pm 0.15$ & $-0.06 \pm 0.06(2)$ \\
\hline YAur & $-0.36 \pm 0.05(6)$ & $-0.30 \pm 0.00(1)$ & $-0.24 \pm 0.10(2)$ & $-0.21 \pm 0.03(2)$ & $-0.24 \pm 0.06(3)$ & $-0.11 \pm 0.00$ \\
\hline YZAur & $-0.34 \pm 0.12(4)$ & $-0.31 \pm 0.00(1)$ & $-0.19 \pm 0.10(5)$ & $0.25 \pm 0.00(1)$ & & $-0.16 \pm 0.00(1)$ \\
\hline
\end{tabular}

